\title{
Efficient Quadrature Solution for Composite Plates with Variable Thickness Resting on Non-Uniform and Nonlinear Elastic Foundation
}

\author{
Ola Ragb*, M. S. Matbuly \\ Department of Physics and Engineering Mathematics, \\ Faculty of Engineering, Zagazig University, \\ P.O. 44519, Zagazig, Egypt
}

\begin{abstract}
Two Different schemes are examined for vibration analysis of composite plate with variable thickness resting on non-uniform and nonlinear elastic foundation problems. On the basis of first order transverse shear theory a basic equation of vibration is derived. Investigations are made over non-uniform and nonlinear Winkler and uniform Pasternak foundation model. Examined schemes are based on discrete singular convolution and moving least square differential quadrature method. Also, the obtained nonlinear algebraic system is solved by using iterative quadrature technique. This problem is solved for different boundary conditions, different shear correction factor and varying thickness in one and two directions. Numerical analysis is applied to investigate influence of different computational characteristics on convergence and accuracy of the obtained results. The obtained results agreed with the previous analytical and numerical ones. Further a parametric study is introduced to explore the influence of elastic and geometric characteristics of the vibrated plate, on results.
\end{abstract}

Keywords-Composite; Vibration; Shear Correction Factor; Non-uniform Elastic Foundation; Nonlinear Winkler Foundation; Variable Thickness; Discrete Singular Convolution; Moving Least Square.

\section{INTRODUCTION}

Non-uniform elastic plates with varying thickness are commonly used in ship and offshore structures, pavement of roads, footing of buildings and bases of machines. Therefore, the vibration analysis of like plates is of great importance for practical design and structural components.

Depending upon the requirement, durability and reliability, materials are being developed so that these may provide better strength, efficiency and economy. Therefore a study of character and behavior of these plates is required so that the full potential of these plates may be used. So, these plates have been studied analytically and numerically. In most cases, their closed form solutions are extremely difficult to establish. But, for some special cases of variable thickness of rectangular plate, investigations have been made and solutions have been obtained [1-4].The most commonly used numerical methods for such applications are Spline function approximation technique [5-6], Rayleigh-Ritz method [7-8], Mesh-free method[9], Mixed boundary node method [10], Finite strip method [11] and Finite element method [12], have been widely applied for the plate resting on uniform elastic foundation. Most of these studies are computationally expensive.

In seeking a more efficient numerical method is differential quadrature method (DQM) which requires fewer grid points yet achieves acceptable accuracy [13-15]. By applying DQM, the free vibration problem of plate is translated into the eigenvalue problem. According to the selection of basis functions and influence domain for each point, there are more than versions of DQM. Discrete singular convolution differential quadrature method (DSCDQM) [16-18], Moving least square differential quadrature method (MLSDQM) [19-21] are the most reliable versions. DSCDQ method is that they exhibit exponential convergence of spectral methods while having the flexibility of local methods for complex boundary conditions. MLSDQ exploits the merits of both the DQ and meshless method.

The main aim of present work to apply two different schemes,( DSCDQM and MLSDQM) to solve vibration problems of composite plates with varying thickness. These plates are resting on non-uniform and nonlinear Winkler and uniform Pasternak elastic foundation. Based on a transverse shear theory, the governing equations of the problem are formulated. The unknown field quantities and their derivatives are approximated using DQ approximations. Then the obtained nonlinear algebraic system is solved by using iterative quadrature technique. The program of MATLAB is designed to solve the reduced eign-value problem. Numerical analysis is implemented to investigate convergence and efficiency of each scheme. Accuracy of the obtained results is compared with the existing previous results. Further a parametric study is introduced to investigate the influence of elastic and geometric characteristics on natural frequencies and mode shapes.

\section{FORMULATION OF THE PROBLEM}

Consider a composite consisting of $n$ plates interfacialy bonded with variable thickness $\mathrm{h}(\mathrm{x}, \mathrm{y})$ and resting on non-uniform and nonlinear elastic foundation of Winkler and uniform Pasternak type, as shown in Fig.(1). 


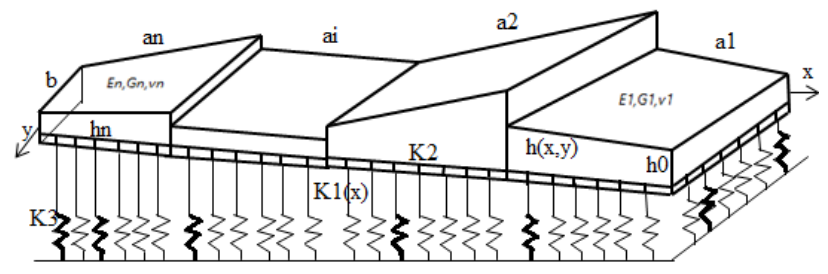

Fig.1 Composite Plate with Variable Thickness resting on Non-uniform and Nonlinear Winkler- Pasternak Foundation.

Each plate occupies $\left(a_{i-1} \leq x \leq a_{i}, \quad 0 \leq y \leq b, \quad i=1, n\right)$, where $\mathrm{a}$ and $\mathrm{b}$ are width and length of the composite. Based on a first-order shear deformation theory, the equations of motion for each plate can be written as [20, 22]: $D(x, y)\left(\frac{\partial^{2} \Phi_{x}}{\partial x^{2}}+\frac{(1-v)}{2} \frac{\partial^{2} \Phi_{x}}{\partial y^{2}}+\frac{(1+v)}{2} \frac{\partial^{2} \Phi_{y}}{\partial x \partial y}\right)+$

$k G h(x, y)\left(\frac{\partial w}{\partial x}-\Phi_{x}\right)=-I_{1} \frac{\partial^{2} \Phi_{x}}{\partial t^{2}}$

$D(x, y)\left(\frac{\partial^{2} \Phi_{y}}{\partial y^{2}}+\frac{(1-v)}{2} \frac{\partial^{2} \Phi_{y}}{\partial x^{2}}+\frac{(1+v)}{2} \frac{\partial^{2} \Phi_{x}}{\partial x \partial y}\right)+$

$k G h(x, y)\left(\frac{\partial w}{\partial y}-\Phi_{y}\right)=-I_{1} \frac{\partial^{2} \Phi_{y}}{\partial t^{2}}$

$k G h(x, y)\left(\frac{\partial^{2} w}{\partial x^{2}}+\frac{\partial^{2} w}{\partial y^{2}}-\frac{\partial \Phi_{x}}{\partial x}-\frac{\partial \Phi_{y}}{\partial y}\right)+$

$K_{1}(x) w+K_{3} w^{3}-K_{2}\left(\frac{\partial^{2} w}{\partial x^{2}}+\frac{\partial^{2} w}{\partial y^{2}}\right)=-I_{0} \frac{\partial^{2} w}{\partial t^{2}}$

$$
I_{0}, I_{1}=\int_{-h_{0} / 2}^{h_{0} / 2}\left(1, z^{2}\right) \rho d z
$$

$\rho$ is the plate mass density. $K_{1}(x)=K_{1}(1+\gamma x / a)$ is the non-uniform Winkler foundation stiffness which linearly varying along $\mathrm{x}$-direction. $\gamma$ is variation parameter of stiffness of foundation. $\mathrm{K}_{2}$ is shear modulus of foundation reaction. $\mathrm{K}_{3}$ is the non-linear Winkler foundation. t: time. $\Phi_{x}(x, y, t), \Phi_{y}(x, y, t), w(x, y, t)$ are normal strain rotations and transverse deflection [24].

The thickness variation function of plate is $h(x, y)=h_{0}(1+\alpha x / a)(1+\beta y / b) . \quad \mathrm{h}_{0}$ is the constant reference thickness value and $\alpha, \beta$ are variation parameter of thickness.
$D(x, y)=E h^{3}(x, y) /\left[12\left(1-v^{2}\right)\right]$ is the flexural rigidity of the plate. G, E and $v$ are shear modulus, Young's modulus and Poisson's ratio of the plate. $\mathrm{k}$ is the shear correction factor [25-27].

Assuming harmonic behavior of the problem, the field quantities can be written as:

$$
\begin{aligned}
& \Phi_{x}(x, y, t)=\varphi_{x} e^{i \omega t} \\
& \Phi_{y}(x, y, t)=\varphi_{y} e^{i \omega t} \\
& w(x, y, t)=W e^{i \omega t}
\end{aligned}
$$

Where $\omega$ is the natural frequency of the plate and $i=\sqrt{-1}$.

$\varphi_{x}, \varphi_{y}, W$ are the amplitudes for $\Phi_{x}, \Phi_{y}$, and $w$, respectively.

Substituting from Eq.(5) into (1-4), One can reduce the problem to:

$$
\begin{aligned}
& D(x, y)\left(\frac{\partial^{2} \varphi_{x}}{\partial x^{2}}+\frac{(1-v)}{2} \frac{\partial^{2} \varphi_{x}}{\partial y^{2}}+\frac{(1+v)}{2} \frac{\partial^{2} \varphi_{y}}{\partial x \partial y}\right)+ \\
& k G h(x, y)\left(\frac{\partial W}{\partial x}-\varphi_{x}\right)=\omega^{2} I_{1} \varphi_{x} \\
& D(x, y)\left(\frac{\partial^{2} \varphi_{y}}{\partial y^{2}}+\frac{(1-v)}{2} \frac{\partial^{2} \varphi_{y}}{\partial x^{2}}+\frac{(1+v)}{2} \frac{\partial^{2} \varphi_{x}}{\partial x \partial y}\right)+ \\
& k G h(x, y)\left(\frac{\partial W}{\partial y}-\varphi_{y}\right)=\omega^{2} I_{1} \varphi_{y},
\end{aligned}
$$

$$
\begin{aligned}
& k G h(x, y)\left(\frac{\partial^{2} W}{\partial x^{2}}+\frac{\partial^{2} W}{\partial y^{2}}-\frac{\partial \varphi_{x}}{\partial x}-\frac{\partial \varphi_{y}}{\partial y}\right)+K_{1}(x) W+K_{3} W^{3} \\
& -K_{2}\left(\frac{\partial^{2} W}{\partial x^{2}}+\frac{\partial^{2} W}{\partial y^{2}}\right)=\omega^{2} I_{0} W
\end{aligned}
$$

Boundary conditions can be expressed as follows:

- Clamped edge:

$$
\begin{aligned}
& W=0, \quad n_{x} \varphi_{y}-n_{y} \varphi_{x}=0, \\
& n_{x} \varphi_{x}+n_{y} \varphi_{y}=0
\end{aligned}
$$


- Simply Supporting of the first kind: SS1

$W=0$,

$$
\begin{aligned}
& \left(n_{x}^{2}+v n_{y}^{2}\right) \frac{\partial \varphi_{x}}{\partial x}+(1-v) n_{x} n_{y} \frac{\partial \varphi_{x}}{\partial y}+ \\
& \left(v n_{x}^{2}+n_{y}^{2}\right) \frac{\partial \varphi_{y}}{\partial y}+(1-v) n_{x} n_{y} \frac{\partial \varphi_{y}}{\partial x}=0 \\
& -\frac{1-v}{2} D\left(\left(n_{x}^{2}-n_{y}^{2}\right) \frac{\partial \varphi_{x}}{\partial y}-2 n_{x} n_{y} \frac{\partial \varphi_{x}}{\partial x}+\right. \\
& \left.\left(n_{x}^{2}-n_{y}^{2}\right) \frac{\partial \varphi_{y}}{\partial x}+2 n_{x} n_{y} \frac{\partial \varphi_{y}}{\partial y}\right)=0
\end{aligned}
$$

- Simply Supporting of the second kind: SS2

$W=0, \quad n_{x} \varphi_{y}-n_{y} \varphi_{x}=0$,

$$
\left(n_{x}^{2}+v n_{y}^{2}\right) \frac{\partial \varphi_{x}}{\partial x}+(1-v) n_{x} n_{y} \frac{\partial \varphi_{x}}{\partial y}+
$$

$$
\left(v n_{x}^{2}+n_{y}^{2}\right) \frac{\partial \varphi_{y}}{\partial y}+(1-v) n_{x} n_{y} \frac{\partial \varphi_{x}}{\partial x}=0
$$

- Free edge:

$k G h\left(n_{x} \frac{\partial W}{\partial x}+n_{y} \frac{\partial W}{\partial y}-n_{x} \varphi_{x}-n_{y} \varphi_{y}\right)=0$

$$
\left(n_{x}^{2}+v n_{y}^{2}\right) \frac{\partial \varphi_{x}}{\partial x}+(1-v) n_{x} n_{y} \frac{\partial \varphi_{x}}{\partial y}+
$$$$
\left(v n_{x}^{2}+n_{y}^{2}\right) \frac{\partial \varphi_{y}}{\partial y}+(1-v) n_{x} n_{y} \frac{\partial \varphi_{y}}{\partial x}=0,
$$

$-\frac{1-v}{2} D\left(\left(n_{x}^{2}-n_{y}^{2}\right) \frac{\partial \varphi_{x}}{\partial y}-2 n_{x} n_{y} \frac{\partial \varphi_{x}}{\partial x}+\right.$ $\left.\left(n_{x}^{2}-n_{y}^{2}\right) \frac{\partial \varphi_{y}}{\partial x}+2 n_{x} n_{y} \frac{\partial \varphi_{y}}{\partial y}\right)=0$

$n_{x}$ and $n_{y}$ are the directional cosines at a point on the boundary edge.

Along the interface between ith plate and $(i+1)$ th one, the continuity boundary conditions can be described as:

$$
\begin{aligned}
& W\left(x, b_{i}^{-}\right)=W\left(x, b_{i}^{+}\right), \quad-D\left(\frac{\partial \varphi_{x}\left(x, b_{i}^{-}\right)}{\partial x}+\right. \\
& \left.v \frac{\partial \varphi_{y}\left(x, b_{i}^{-}\right)}{\partial y}\right)=-D\left(\frac{\partial \varphi_{x}\left(x, b_{i}^{+}\right)}{\partial x}+v \frac{\partial \varphi_{y}\left(x, b_{i}^{+}\right)}{\partial y}\right), \\
& \frac{1-v}{2}\left(\frac{\partial \varphi_{x}\left(x, b_{i}^{-}\right)}{\partial y}+\frac{\partial \varphi_{y}\left(x, b_{i}^{-}\right)}{\partial x}\right)= \\
& \frac{1-v}{2}\left(\frac{\partial \varphi_{x}\left(x, b_{i}^{+}\right)}{\partial y}+\frac{\partial \varphi_{y}\left(x, b_{i}^{+}\right)}{\partial x}\right), \quad(i=1, n)
\end{aligned}
$$

\section{SOLUTION OF THE PROBLEM}

Two different differential quadrature techniques are employed to reduce the governing equations into nonlinear eigenvalue problem, as follows [16-21]:

- Discrete Singular Convolution Differential Quadrature Method (DSCDQM)

In this technique, regularized Shannon kernel (RSK) may be used as a shape function such that the unknown $\mathrm{u}(\mathrm{x})$ and its derivatives can be approximated over a narrow bandwidth

$$
\begin{gathered}
\left(x-x_{M}, x+x_{M}\right) \text { as [16-18]: } \\
u\left(x_{i}\right)=\sum_{j=-M}^{M}\left\langle\frac{\sin \left[\pi\left(x_{i}-x_{j}\right) / h_{x}\right]}{\pi\left(x_{i}-x_{j}\right) / h_{x}} e^{-\left(\frac{\left(x_{i}-x_{j}\right)^{2}}{2 \sigma^{2}}\right)}\right\rangle u\left(x_{j}\right),
\end{gathered}
$$

$$
(i=-N, N)
$$

where $h_{x}$ is the step size, $2 M+1$ is the effective computational band width, $\sigma$ is regularization parameter, $\sigma$ $=r h x$ and $r$ is a computational parameter.

Derivatives of $u$, can be approximated as a weighted linear sum of $u_{i},(i=-N, N)$ as [16-18]:

$$
\begin{aligned}
& \left.\frac{\partial u}{\partial x}\right|_{x=x_{i}}=\sum_{j=-M}^{M} C_{i j}^{x} u\left(x_{j}\right), \\
& \left.\frac{\partial^{2} u}{\partial x^{2}}\right|_{x=x_{i}}=\sum_{j=-M}^{M} C_{i j}^{x x} u\left(x_{j}\right), \quad(i=-N, N),
\end{aligned}
$$

Where

$$
C_{i j}^{x}=\left\{\begin{array}{ll}
\frac{(-1)^{i-j}}{h_{x}(i-j)} e^{-h_{x}\left(\frac{(i-j)^{2}}{2 \sigma^{2}}\right)}, & i \neq j \\
0 & i=j
\end{array},\right.
$$


$C_{i j}^{x x}= \begin{cases}\left(\frac{2(-1)^{i-j+1}}{h_{x}^{2}(i-j)^{2}}+\frac{1}{\sigma^{2}}\right) e^{-h_{x}^{2}\left(\frac{(i-j)^{2}}{2 \sigma^{2}}\right)}, & i \neq j \\ -\frac{1}{\sigma^{2}}-\frac{\pi^{2}}{3 h_{x}^{2}} & i=j\end{cases}$

Similarly, one can approximate $u_{y}, u_{y y}$ and calculated $C_{i j}^{y}, C_{i j}^{y y}$.

\section{- Moving Least Squares Differential Quadrature Method} (MLSDQM)

In this technique, the influence domain, $\left(\Omega_{\mathrm{i}}, \mathrm{i}=1\right.$ : $\left.N\right)$, for each node is determined as shown in Fig. (2). Over each influence domain, the nodal unknowns can be approximated as [19-21]:

$u\left(\mathbf{x}_{i}\right) \cong u^{h}\left(\mathbf{x}_{i}\right)=\sum_{j=1}^{N_{1}} \phi_{j}\left(\mathbf{x}_{i}\right) u\left(\mathbf{x}_{j}\right),(i=1, N)$,

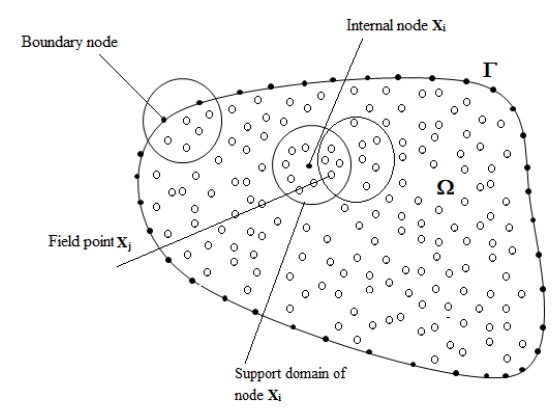

Fig.2 Domain discretization for moving least squares differential quadrature.

Where the shape function $\phi_{j}\left(\mathbf{x}_{i}\right)$ can be obtained using MLS approximation as follows:

$$
\text { Let } \quad u^{h}(\mathbf{x})=\sum_{i=1}^{m} P_{i}(\mathbf{x}) a_{i}(\mathbf{x})=P^{T}(\mathbf{x}) a(\mathbf{x}),
$$

Where $a(\mathbf{x})=\left\{a_{1}(\mathbf{x}), a_{2}(\mathbf{x}), \cdots, a_{m}(\mathbf{x})\right\}^{T}$ is a vector of unknown coefficients.

$P^{T}(\mathbf{x})=\left\{p_{1}(\mathbf{x}), p_{2}(\mathbf{x}), \cdots, p_{m}(\mathbf{x})\right\}$ is a complete set of monomial basis. $\mathrm{m}$ is the number of basis terms. The coefficients $a_{j}(\mathbf{x}),(j=1, m)$, can be obtained at any point $\mathrm{x}$ by minimizing the following weighted quadratic for

$$
\begin{aligned}
& \Pi(\mathbf{a})=\sum_{i=1}^{N_{1}} \varpi\left(\mathbf{x}-\mathbf{x}_{i}\right)\left(u^{h}\left(\mathbf{x}_{i}\right)-u_{i}\right)^{2}= \\
& \sum_{i=1}^{N_{1}} \varpi\left(\mathbf{x}-\mathbf{x}_{i}\right)\left(\mathbf{P}^{T}\left(\mathbf{x}_{i}\right) \mathbf{a}(\mathbf{x})-u_{i}\right)^{2}
\end{aligned}
$$

Where $\varpi(\mathrm{x}-\mathrm{xi})$ is a positive weight function defined over the influence domain, $\left(\Omega_{\mathrm{i}}, \mathrm{i}=1, \mathrm{~N}\right)$

The stationary value of $\Pi(a)$ with respect to $a(x)$ leads to a linear equation, such as:

$$
\mathbf{A}(\mathbf{x}) \mathbf{a}(\mathbf{x})=\mathbf{B}(\mathbf{x}) \mathbf{u}
$$

from which $\mathbf{a}(\mathbf{x})=\mathbf{A}^{-1}(\mathbf{x}) \mathbf{B}(\mathbf{x}) \mathbf{u}$,

where

$$
\begin{aligned}
& \mathbf{A}(\mathbf{x})=\mathbf{P}\left(\mathbf{x}_{i}\right) \varpi_{i}(\mathbf{x}) \mathbf{P}^{T}\left(\mathbf{x}_{i}\right)= \\
& \sum_{i=1}^{N_{1}} \varpi_{i}(\mathbf{x}) \mathbf{P}\left(\mathbf{x}_{i}\right) \mathbf{P}^{T}\left(\mathbf{x}_{i}\right), \quad \mathbf{u}=\left[\begin{array}{llll}
u_{1} & u_{2} & \cdots & u_{n}
\end{array}\right]^{T}, \\
& \mathbf{B}(\mathbf{x})=\mathbf{P}(\mathbf{x}) \varpi(\mathbf{x})= \\
& {\left[\begin{array}{llll}
\varpi_{1}(\mathbf{x}) \mathbf{P}\left(\mathbf{x}_{1}\right) & \varpi_{2}(\mathbf{x}) \mathbf{P}\left(\mathbf{x}_{2}\right) & \cdots & \varpi_{n}(\mathbf{x}) \mathbf{P}\left(\mathbf{x}_{n}\right)
\end{array}\right]}
\end{aligned}
$$

On suitable substitution from Eq. (22) into (19), $\mathrm{u}^{\mathrm{h}}(\mathrm{x})$ can then be expressed as:

$$
\begin{aligned}
& u^{h}(\mathbf{x})=\mathbf{P}^{T}(\mathbf{x}) \mathbf{a}(\mathbf{x})= \\
& \mathbf{P}^{T}(\mathbf{x}) \mathbf{A}^{-1}(\mathbf{x}) \mathbf{B}_{\mathbf{i}}(\mathbf{x}) \mathbf{u}=\sum_{i=1}^{N_{1}} \phi_{i}(\mathbf{x}) u_{i}
\end{aligned}
$$

Where the nodal shape function:

$$
\phi_{i}(\mathbf{x})=\mathbf{P}^{T}(\mathbf{x}) \mathbf{A}^{-1}(\mathbf{x}) \mathbf{B}_{\mathbf{i}}(\mathbf{x})
$$

Determination of the shape function $\phi_{i}(\mathbf{x})$ and its partial derivatives can be simplified as follows [19-21]:

$\phi_{i}(\mathbf{x})=\mathrm{P}^{\mathrm{T}}(\mathbf{x}) \mathrm{A}^{-1}(\mathbf{x}) \mathrm{B}_{\mathrm{i}}(\mathbf{x})=\alpha^{T}(\mathbf{x}) \mathrm{B}_{\mathrm{i}}(\mathbf{x})$

Since $A(x)$ is a symmetric matrix, then $\alpha(\mathbf{x})$ can be obtained through

$$
\mathbf{A}(\mathbf{x}) \alpha(\mathbf{x})=\mathbf{P}(\mathbf{x})
$$

Therefore, the problem of determination of the shape function is reduced to solution of Eq.(26). This Equation can be solved using LU decomposition and back-substitution, which requires fewer computations than the inversion of 
$A(x)$. Further, the first and second order partial derivatives of $\phi_{j}\left(\mathbf{x}_{i}\right)$ can be determined as follows [19-21]:

Differentiate Eq. (26) with respect to $\mathrm{L}, \mathrm{K},(\mathrm{L}, \mathrm{K}=\mathrm{X}, \mathrm{Y}$ ) such as:

$$
\begin{aligned}
& \mathbf{A}(\mathbf{x}) \alpha_{, L}(\mathbf{x})=\mathbf{P}_{, L}(\mathbf{x})-\mathbf{A}_{, L}(\mathbf{x}) \alpha(\mathbf{x}), \\
& (L=X, Y)
\end{aligned}
$$

$$
\begin{aligned}
& \mathrm{A}(\mathbf{x}) \alpha_{, L K}(\mathbf{x})=\mathrm{P}_{, L K}(\mathbf{x})-\mathrm{A}_{, L K}(\mathbf{x}) \alpha(\mathbf{x})- \\
& \mathrm{A}_{, L}(\mathbf{x}) \alpha_{, K}(\mathbf{x})-\mathrm{A}_{, K}(\mathbf{x}) \alpha_{, L}(\mathbf{x}),(L, \mathrm{~K}=X, Y)
\end{aligned}
$$

The first and second order partial derivatives of the shape function can be described as:

$$
\begin{aligned}
& \phi_{j, L}\left(\mathbf{x}_{i}\right)=c_{j}^{L}\left(\mathbf{x}_{i}\right)=\alpha_{j, L}^{T}\left(\mathbf{x}_{i}\right) \mathrm{B}_{j}\left(\mathbf{x}_{i}\right)+ \\
& \alpha_{j}^{T}\left(\mathbf{x}_{i}\right) \mathrm{B}_{j, L}\left(\mathbf{x}_{i}\right), \quad(L=x, y), \\
& \phi_{j, L K}\left(\mathbf{x}_{i}\right)=c_{j}^{L K}\left(\mathbf{x}_{i}\right)=\alpha_{j, L K}^{T}\left(\mathbf{x}_{i}\right) \mathrm{B}_{j}\left(\mathbf{x}_{i}\right)+ \\
& \alpha_{j}^{T}\left(\mathbf{x}_{i}\right) \mathrm{B}_{j, L K}\left(\mathbf{x}_{i}\right)+\alpha_{j, L}^{T}\left(\mathbf{x}_{i}\right) \mathrm{B}_{j, K}\left(\mathbf{x}_{i}\right)+\alpha_{j, K}^{T}\left(\mathbf{x}_{i}\right) \mathrm{B}_{j, L}\left(\mathbf{x}_{i}\right), \\
& (L, K=x, y)
\end{aligned}
$$

On suitable substitution from Eqs.(14-30) into (6-8), the problem can be reduced to the following nonlinear eigenvalue problem:

$\sum_{j=1}^{N}\left[\begin{array}{l}\left.k G h^{i j} c_{i j}^{x} W^{j}+D^{i j}\left(c_{i j}^{x x}+\frac{1-v}{2} c_{i j}^{y y}-k G h^{i j}\right) \varphi_{x}^{j}+\right] \\ D^{i j}\left(\frac{1+v}{2} c_{i k}^{x} c_{k j}^{y}\right) \varphi_{y}^{j}\end{array}\right]=$
$\omega^{2} I_{1} \varphi_{x}^{j},(i, k=1, N)$

$\sum_{j=1}^{N}\left[\begin{array}{l}k G h^{i j} c_{i j}^{y} W^{j}+D^{i j}\left(\frac{1+v}{2} c_{i k}^{x} c_{k j}^{y}\right) \varphi_{x}^{j}+ \\ D^{i j}\left(c_{i j}^{y y}+\frac{1-v}{2} c_{i j}^{x x}-k G h^{i j}\right) \varphi_{y}^{j}\end{array}\right]=$

$\omega^{2} I_{1} \varphi_{y}^{j},(i, k=1, N)$

$\sum_{j=1}^{N} k G h^{i j}\left[\left(c_{i j}^{x x}+c_{i j}^{y y}\right) W^{j}-c_{i j}^{x} \varphi_{x}^{j}-c_{i j}^{y} \varphi_{y}^{j}\right]-$

$K_{2}\left(c_{i j}^{x x}+c_{i j}^{y y}\right) W^{j}+K_{1}^{i} W^{j}+K_{3}\left(W^{2}\right) W^{j}=$

$$
\omega^{2} I_{0} W^{j},(i=1, N)
$$

The boundary conditions (9-13) can also be approximated using DQMs as:

$$
\text { - Clamped edge: }
$$

$$
\begin{aligned}
& W^{i}=0, \quad n_{x} \varphi_{y}^{i}-n_{y} \varphi_{x}^{i}=0, \quad n_{x} \varphi_{x}^{i}+ \\
& n_{y} \varphi_{y}^{i}=0, \quad(i=1, N)
\end{aligned}
$$

- Simply supporting of the first kind: SS1

$$
\begin{aligned}
& W^{i}=0, \\
& \sum_{j=1}^{N}\left[\begin{array}{l}
\left(\left(n_{x}^{2}+v n_{y}^{2}\right) c_{i j}^{x}+(1-v) n_{x} n_{y} c_{i j}^{y}\right) \varphi_{x}^{j}+ \\
\left.\left(v n_{x}^{2}+n_{y}^{2}\right) c_{i j}^{y}+(1-v) n_{x} n_{y} c_{i j}^{x}\right) \varphi_{y}^{j}
\end{array}\right]=0, \\
& -\frac{1-v}{2} D^{i j} \sum_{j=1}^{N}\left[\begin{array}{l}
\left(\left(n_{x}^{2}-n_{y}^{2}\right) c_{i j}^{y}-2 n_{x} n_{y} c_{i j}^{x}\right) \varphi_{x}^{j}+ \\
\left(\left(n_{x}^{2}-n_{y}^{2}\right) c_{i j}^{x}+2 n_{x} n_{y} c_{i j}^{y}\right) \varphi_{y}^{j}
\end{array}\right]=0, \\
& (i=1, N)
\end{aligned}
$$

- Simply supporting of the second kind: SS2

$$
\begin{aligned}
& W^{i}=0, \quad n_{x} \varphi_{y}^{i}-n_{y} \varphi_{x}^{i}=0, \\
& \sum_{j=1}^{N}\left[\begin{array}{l}
\left(\left(n_{x}^{2}+v n_{y}^{2}\right) c_{i j}^{x}+(1-v) n_{x} n_{y} c_{i j}^{y}\right) \varphi_{x}^{j}+ \\
\left(\left(v n_{x}^{2}+n_{y}^{2}\right) c_{i j}^{y}+(1-v) n_{x} n_{y} c_{i j}^{x}\right) \varphi_{y}^{j}
\end{array}\right]=0 \\
& (i=1, N)
\end{aligned}
$$

- Free edge:

$k G h^{i j}\left(\sum_{j=1}^{N}\left(n_{x} c_{i j}^{x}+n_{y} c_{i j}^{y}\right) W^{j}\right)-k G h^{i j}\left(n_{x} \varphi_{x}^{i}+n_{y} \varphi_{y}^{i}\right)=0$,

$\sum_{j=1}^{N}\left[\begin{array}{l}\left(\left(n_{x}^{2}+v n_{y}^{2}\right) c_{i j}^{x}+(1-v) n_{x} n_{y} c_{i j}^{y}\right) \varphi_{x}^{j}+ \\ \left(\left(v n_{x}^{2}+n_{y}^{2}\right) c_{i j}^{y}+(1-v) n_{x} n_{y} c_{i j}^{x}\right) \varphi_{y}^{j}\end{array}\right]=0$,
$-\frac{1-v}{2} D^{i j} \sum_{j=1}^{N}\left[\begin{array}{l}\left(\left(n_{x}^{2}-n_{y}^{2}\right) c_{i j}^{y}-2 n_{x} n_{y} c_{i j}^{x}\right) \varphi_{x}^{j}+ \\ \left(\left(n_{x}^{2}-n_{y}^{2}\right) c_{i j}^{x}+2 n_{x} n_{y} c_{i j}^{y}\right) \varphi_{y}^{j}\end{array}\right]=0$

\section{NUMERICAL RESULTS}

This section presents numerical results that demonstrate convergence and efficiency of each one of the proposed schemes for vibration analysis of nonuniform and nonlinear Winkler and uniform Pasternak foundation of composite plate. This plate varying with thickness. For all results, the boundary conditions (34-37) are augmented in the governing equations (31-33). Then the obtained nonlinear algebraic system is solved by using iterative quadrature technique [34]. The computational characteristics of each scheme are adapted to reach accurate results with error of order $\leq 10^{-10}$. The obtained frequencies $\omega$ are normalized such as: $\Omega=(\omega \sqrt{I}) /\left(\pi^{2} \Omega_{0}\right)$ where $\Omega_{0}$ is the fundamental frequency of isotropic squared plate.

For DSCDQ scheme based on regularized Shannon kernel (RSK), the problem is also solved over a uniform grids ranging from $7 * 7$ to $19 * 19$. The bandwidth $2 \mathrm{M}+1$ 
ranges from 5 to 15 and the regularization parameter $\sigma=r h_{x}$ ranges from $1.5 h_{x}$ to $3 h_{x}$, where $h_{x}=1 / N-1$. Table (1) shows convergence of the obtained fundamental frequency to the exact and numerical ones [28-31] over grid size $\geq 13 * 13$, bandwidth $\geq 11$ and regulization parameter $\sigma=2.82 \mathrm{~h}_{\mathrm{x}}$.

TABLE. 1 Comparison between the fundamental frequency due to DSCDQM-RSK with the bandwidth $2 \mathrm{M}+1$ ,regularization parameter $\sigma$, the grid points $\mathrm{N}$ and the previous results for a regular discretized isotropic simply supported squared plate: $\left(\mathrm{h}_{0} / \mathrm{a}=0.01, \mathrm{v}=0.3\right.$, $\mathrm{k}=5 / 6, \mathrm{~K} 1=500, \alpha=0, \beta=0, \gamma=0, \mathrm{~K} 2=0, \mathrm{~K} 3=0$ ).

\begin{tabular}{|c|c|c|c|c|c|c|c|}
\hline \multirow{2}{*}{$\begin{array}{l}\text { fundamental } \\
\text { frequency }\end{array}$} & \multicolumn{2}{|c|}{ Number of grid points } & \multirow{2}{*}{9} & \multirow{2}{*}{11} & \multirow{2}{*}{13} & \multirow{2}{*}{15} & \multirow{2}{*}{17} \\
\hline & $\begin{array}{l}\text { regularization } \\
\text { parameter }\end{array}$ & $\begin{array}{l}\text { Band } \\
\text { width }\end{array}$ & & & & & \\
\hline \multirow{16}{*}{$\underset{R S K}{D S C D Q M-}$} & \multirow{4}{*}{$\sigma=1.8 h_{x}$} & $2 M+1=7$ & 2.03 & 2.46 & 2.74 & 2.89 & 2.9545 \\
\hline & & $2 M+1=9$ & 2.23 & 2.69 & 2.82 & 2.93 & 2.9736 \\
\hline & & $2 M+1=11$ & 2.58 & 2.88 & 2.91 & 2.95 & 2.9828 \\
\hline & & $2 M+1=13$ & 2.79 & 2.92 & 2.96 & 2.99 & 2.9991 \\
\hline & \multirow{4}{*}{$\sigma=2.4 h_{x}$} & $2 M+1=7$ & 2.29 & 2.64 & 2.83 & 2.92 & 2.9683 \\
\hline & & $2 M+1=9$ & 2.52 & 2.72 & 2.88 & 2.96 & 2.9743 \\
\hline & & $2 M+1=11$ & 2.61 & 2.91 & 2.94 & 2.98 & 2.9915 \\
\hline & & $2 M+1=13$ & 2.86 & 2.95 & 2.99 & 2.99 & 2.9976 \\
\hline & \multirow{4}{*}{$\sigma=2.62 h_{x}$} & $2 M+1=7$ & 2.58 & 2.72 & 2.89 & 2.96 & 2.9697 \\
\hline & & $2 M+1=9$ & 2.70 & 2.85 & 2.94 & 2.99 & 2.9953 \\
\hline & & $2 M+1=11$ & 2.76 & 2.94 & 2.99 & 3.01 & 3.0224 \\
\hline & & $2 M+1=13$ & 2.89 & 2.98 & 3.01 & 3.02 & 3.0213 \\
\hline & \multirow{4}{*}{$\sigma=2.82 h_{x}$} & $2 M+1=7$ & 2.86 & 2.92 & 2.98 & 2.99 & 3.0026 \\
\hline & & $2 M+1=9$ & 2.89 & 2.98 & 2.99 & 3.005 & 3.0141 \\
\hline & & $2 M+1=11$ & 2.94 & 2.99 & 3.02 & 3.022 & 3.0215 \\
\hline & & $2 M+1=13$ & 2.97 & 3.01 & 3.02 & 3.022 & 3.0215 \\
\hline \multicolumn{3}{|c|}{ Exact results [28] } & \multicolumn{5}{|c|}{3.0215} \\
\hline \multicolumn{3}{|c|}{ Ritz method[29] } & & & \multicolumn{3}{|c|}{3.0214} \\
\hline \multicolumn{3}{|c|}{ Radial basis[30] } & & & \multicolumn{3}{|c|}{3.0216} \\
\hline \multicolumn{3}{|c|}{ Element free Galerkin[31] } & & & \multicolumn{3}{|c|}{3.0225} \\
\hline
\end{tabular}

For MLSDQ scheme, circular influence domain, $(\Omega \mathrm{i}, \mathrm{i}=1, \mathrm{~N})$, is considered as shown in Fig.2. Gaussian weight function is employed such as [19-21]:

$w_{i}(x)= \begin{cases}\frac{\exp \left(-\left(d_{i} / c\right)^{2}\right)-\exp \left(-(r / c)^{2}\right)}{1-\exp \left(-(r / c)^{2}\right)} & d_{i} \leq r \\ 0 & d_{i}>r\end{cases}$

Where $d_{i}$ is the distance from a nodal point $\mathrm{x}_{\mathrm{i}}$ to a field one $\mathrm{x}$ located in the influence domain of $\mathrm{x}_{\mathrm{i}} . \mathrm{r}$ is the radius of support domain and $\mathrm{c}$ is the dilation parameter. In the present work, the dilation parameter is selected such as: $c=r / 4$.

The scheme is employed with varying completeness order $\mathrm{N}_{\mathrm{c}}$ ranges from 2 to 5 and the raduis of support domain $d_{\max }=r / h_{x}$ ranges from 4 to 9 . Also, the problem is also solved over a uniform grids ranging from $5 * 5$ to $19 * 19$. Table (2) shows convergence of the obtained fundamental frequency to the exact and numerical ones [28-31] over grid size $\geq 11 * 11$, completeness order $\geq 4$ and raduis of support domain $\mathrm{d}_{\max }=5$.

TABLE.2 Comparison between the fundamental frequency due to MLSDQM with the radius of support domain $d_{\max }$ completeness order $\mathrm{N}_{\mathrm{c}}$, the grid points $\mathrm{N}$ and the previous results for a regular discretized isotropic simply supported squared plate: $\left(\mathrm{h} / \mathrm{a}=0.01, \mathrm{v}=0.3, \mathrm{k}=5 / 6, \mathrm{~K}_{1}=100, \alpha=0, \beta=0, \gamma=0\right.$, $\mathrm{K}_{2}=0, \mathrm{~K}_{3}=0$ ).

\begin{tabular}{|c|c|c|c|c|c|c|}
\hline \multirow{2}{*}{$\begin{array}{l}\text { fundamental } \\
\text { frequency }\end{array}$} & \multicolumn{2}{|c|}{$\begin{array}{l}\text { Number of grid } \\
\text { points }\end{array}$} & \multirow{2}{*}{7} & \multirow{2}{*}{9} & \multirow{2}{*}{11} & \multirow{2}{*}{13} \\
\hline & $\begin{array}{l}\text { completeness } \\
\text { order }\end{array}$ & $\begin{array}{l}\text { Radius of } \\
\text { support } \\
\text { domain }\end{array}$ & & & & \\
\hline \multirow{16}{*}{$M L S D Q M$} & \multirow{4}{*}{$N_{c}=2$} & $d_{\max }=5$ & 2.64 & 2.516 & 2.446 & 2.4509 \\
\hline & & $d_{\max }=6$ & 2.31 & 2.295 & 2.272 & 2.2612 \\
\hline & & $d_{\max }=7$ & 2.26 & 2.254 & 2.251 & 2.2491 \\
\hline & & $d_{\max }=8$ & 2.25 & 2.247 & 2.246 & 2.2436 \\
\hline & \multirow{4}{*}{$N_{c}=3$} & $d_{\max }=5$ & 2.25 & 2.245 & 2.245 & 2.2441 \\
\hline & & $d_{\max }=6$ & 2.25 & 2.243 & 2.243 & 2.2421 \\
\hline & & $d_{\max }=7$ & 2.24 & 2.242 & 2.242 & 2.2414 \\
\hline & & $d_{\max }=8$ & 2.24 & 2.241 & 2.241 & 2.2413 \\
\hline & \multirow{4}{*}{$N_{c}=4$} & $d_{\max }=5$ & 2.24 & 2.241 & 2.2413 & 2.2413 \\
\hline & & $d_{\max }=6$ & 2.24 & 2.241 & 2.2413 & 2.2413 \\
\hline & & $d_{\max }=7$ & 2.24 & 2.241 & 2.2413 & 2.2413 \\
\hline & & $d_{\max }=8$ & 2.24 & 2.241 & 2.2413 & 2.2413 \\
\hline & \multirow{4}{*}{$N_{c}=5$} & $d_{\max }=5$ & 2.74 & 2.244 & 2.2413 & 2.2413 \\
\hline & & $d_{\max }=6$ & 2.25 & 2.249 & 2.2413 & 2.2413 \\
\hline & & $d_{\max }=7$ & 2.26 & 2.241 & 2.2413 & 2.2413 \\
\hline & & $d_{\max }=8$ & 2.26 & 2.241 & 2.2413 & 2.2413 \\
\hline \multicolumn{3}{|c|}{ Exact results [28] } & \multicolumn{4}{|c|}{2.2413} \\
\hline \multicolumn{3}{|c|}{ Ritz method[29] } & \multicolumn{4}{|c|}{2.2413} \\
\hline \multicolumn{3}{|c|}{ Radial basis[30] } & \multicolumn{4}{|c|}{2.2414} \\
\hline \multicolumn{3}{|c|}{$\begin{array}{l}\text { Element free Galerkin[31] } \\
(15 \times 15)\end{array}$} & \multicolumn{4}{|c|}{2.2427} \\
\hline
\end{tabular}

Table (3) shows that the best value of Shear factor correction is to be taken $(5 / 6-v)$. Tables $(3,4)$ show that the fundamental frequency decrease with increasing Poisson ratio and Shear factor correction. Also ,the value of shear factor correction helps to achieve more accurate results.

TABLE.3 Comparison between the fundamental frequency, Poisson, shear factor correction $\mathrm{K}$ and the previous results for CCCS squared plate: $\left(\mathrm{h}_{0} / \mathrm{a}=0.01, \mathrm{~K}_{1}=0, \alpha=0.5, \beta=0.5, \gamma=0\right.$, $\mathrm{K}_{2}=0, \mathrm{~K}_{3}=0, \mathrm{E}_{1} / \mathrm{E}_{2}=2.45$ ).

\begin{tabular}{|c|c|c|c|c|c|c|}
\hline $\begin{array}{l}\text { Poisson } \\
\text { ratio v }\end{array}$ & $\begin{array}{l}\text { Discrete } \\
\text { Green } \\
\text { function } \\
{[33]}\end{array}$ & $\begin{array}{l}k= \\
\frac{5}{6-v}\end{array}$ & $\begin{array}{l}k=\frac{5}{6} * \\
\frac{\left(1+\pi^{2}\left(h_{0} / a\right)^{2}\right)}{\left(210\left(1-v^{2}\right)\right)}\end{array}$ & $\begin{array}{l}k=\frac{5}{6} * \\
\frac{\left(1+\pi^{2}\left(h_{0} / a\right)^{2}\right)}{\left(210 a^{2}\left(1-v^{2}\right)\right)}\end{array}$ & $\begin{array}{l}k= \\
\frac{5}{6}\end{array}$ & $\begin{array}{l}k= \\
\frac{\pi^{2}}{12}\end{array}$ \\
\hline 0.15 & & 8.1424 & 8.1638 & 8.1639 & 8.164 & 8.163 \\
\hline 0.23 & 8.1110 & 8.1110 & 8.1112 & 8.1112 & 8.111 & 8.111 \\
\hline 0.3 & & 8.0640 & 8.04586 & 8.0460 & 8.046 & 8.046 \\
\hline 0.5 & - & 7.8090 & 7.7430 & 7.7432 & 7.743 & 7.744 \\
\hline
\end{tabular}


TABLE.4 Comparison between the fundamental frequency, Poisson, shear factor correction $\mathrm{K}$ and different boundary condition for squared plate: $\left(\mathrm{h}_{0} / \mathrm{a}=0.01, \mathrm{~K}_{1}=100, \alpha=0, \beta=0, \gamma=0\right.$, $\mathrm{K}_{2}=50, \mathrm{~K}_{3}=0, \mathrm{E}_{1} / \mathrm{E}_{2}=2.45$ ).

\begin{tabular}{|c|l|l|l|l|l|}
\hline $\begin{array}{c}\text { Poisson } \\
\text { ratio } \boldsymbol{v}\end{array}$ & $\begin{array}{l}\text { shear factor } \\
\text { correction } \\
\boldsymbol{k}=\frac{\mathbf{5}}{\mathbf{6 - \boldsymbol { v }}}\end{array}$ & SSSS & CCCC & CSSS & CCSC \\
\hline 0.15 & 0.8547 & 3.9382 & 5.20912 & 4.2312 & 4.87028 \\
\hline 0.23 & 0.8666 & 3.9207 & 5.18596 & 4.2123 & 4.84863 \\
\hline 0.3 & 0.8772 & 3.8935 & 5.1501 & 4.1831 & 4.8151 \\
\hline 0.5 & 0.9091 & 3.7429 & 4.95105 & 4.0213 & 4.62899 \\
\hline
\end{tabular}

Tables $(5,6)$ show that execution time of DSCDQM-RSK scheme is less than that of MLSDQM. Therefore, it is more efficient than MLSDQM for vibration analysis of variable thickness, non-uniform and nonlinear elastically supported composite plate.

TABLE. 5 Comparison between the obtained natural frequencies and the previous results for isotropic clamped plate: $\left(\mathrm{h}_{0} / \mathrm{a}=0.015, v=0.15, \alpha=0, \beta=0, \gamma=0, \mathrm{~K}_{2}=0, \mathrm{~K}_{3}=0\right)$.

\begin{tabular}{|c|c|c|c|c|c|c|c|}
\hline $\begin{array}{l}\text { Subgrade } \\
\text { reaction }\end{array}$ & \multicolumn{3}{|c|}{$K_{1}=1390.2$} & \multicolumn{3}{|c|}{$K_{1}=2780.4$} & \multirow{2}{*}{$\begin{array}{l}\text { Execution } \\
\text { time }(\text { sec })\end{array}$} \\
\hline Results & & $\overline{\omega_{2}}$ & $\overline{\omega_{3}}$ & $\overline{\omega_{1}}$ & $\overline{\omega_{2}}$ & $\overline{\omega_{3}}$ & \\
\hline $\begin{array}{l}\text { MLSDQM }(11 x 11), \\
N_{c}=4 \text { and } d_{\max }=5\end{array}$ & 5.245 & 8.316 & 8.316 & 6.463 & 9.132 & 9.132 & 1.676119 \\
\hline $\begin{array}{l}D S C D Q M- \\
R S K(13 x 13) \\
\sigma=2.82 h x \text { and } \\
M=5\end{array}$ & 5.245 & 8.316 & 8.316 & 6.463 & 9.132 & 9.132 & 1.656792 \\
\hline $\begin{array}{l}\text { Mixed finite } \\
\text { element [32] }\end{array}$ & 5.245 & 8.316 & 8.316 & 6.463 & 9.132 & 9.132 & \\
\hline $\begin{array}{c}\text { Element free } \\
\text { Galerkin[31] }\end{array}$ & 5.267 & 8.391 & 8.392 & 6.477 & 9.202 & 9.203 & \\
\hline Ritz method[29] & 5.259 & 8.432 & 8.432 & 6.460 & 9.248 & 9.248 & \\
\hline Radial basis[30 ] & 5.244 & 8.313 & 8.313 & 6.463 & 9.130 & 9.130 & \\
\hline
\end{tabular}

Also, for different boundary conditions and varying thickness in one and two directions, tables $(6,7)$ insist that DSCDQM-RSK scheme is the best choice for such problem.

TABLE. 6 Comparison between the natural frequency due to DSCDQM-RSK and MLSDQM with the variable thickness $\alpha$ in one direction, different boundary conditions and the previous results for a squared plate: $\left(\mathrm{h} / \mathrm{a}=0.01, \mathrm{E}_{1} / \mathrm{E}_{2}=2.45\right.$ $\left.v=0.23, \mathrm{~K}_{1}=0, \beta=0, \mathrm{~K}_{2}=0, \mathrm{~K}_{3}=0\right)$.

\begin{tabular}{|c|c|c|c|c|c|c|}
\hline \multirow[b]{2}{*}{$\begin{array}{l}\text { Boundary } \\
\text { coditions }\end{array}$} & \multicolumn{2}{|c|}{ natural frequency } & \multirow[b]{2}{*}{$\overline{\omega_{1}}$} & \multirow[b]{2}{*}{$\overline{\omega_{2}}$} & \multirow[b]{2}{*}{$\overline{\omega_{3}}$} & \multirow{2}{*}{$\begin{array}{l}\text { Execution } \\
\text { time (sec) }\end{array}$} \\
\hline & $\begin{array}{l}\text { variable } \\
\text { thickness }\end{array}$ & results & & & & \\
\hline \multirow{12}{*}{ SSSS } & \multirow{4}{*}{$\alpha=0$} & $\begin{array}{l}\text { DSCDQM- } \\
\text { RSK }\end{array}$ & 4.902 & 7.253 & 8.374 & 1.780280 \\
\hline & & $M L S D Q M$ & 4.902 & 7.253 & 8.374 & 1.839144 \\
\hline & & $\begin{array}{l}\text { Element free } \\
\text { Galerkin } \\
{[31]}\end{array}$ & 4.900 & 7.256 & 8.382 & \\
\hline & & $\begin{array}{l}\text { Discrete } \\
\text { Green } \\
\text { function [33] }\end{array}$ & 4.902 & 7.253 & 8.374 & \\
\hline & \multirow{4}{*}{$\alpha=0.4$} & $\begin{array}{l}\text { DSCDQM- } \\
\text { RSK }\end{array}$ & 5.360 & 7.928 & 9.150 & 1.889903 \\
\hline & & $M L S D Q M$ & 5.360 & 7.928 & 9.150 & 1.918841 \\
\hline & & $\begin{array}{l}\text { Element free } \\
\text { Galerkin } \\
{[31]} \\
31]\end{array}$ & 5.356 & 7.931 & 9.159 & \\
\hline & & $\begin{array}{l}\text { Discrete } \\
\text { Green } \\
\text { function [33] }\end{array}$ & 5.360 & 7.928 & 9.150 & \\
\hline & \multirow{4}{*}{$\alpha=0.8$} & $\begin{array}{l}\text { DSCDQM- } \\
\text { RSK }\end{array}$ & 5.770 & 8.525 & 9.831 & 1.900178 \\
\hline & & $M L S D Q M$ & 5.770 & 8.525 & 9.831 & 1.928867 \\
\hline & & $\begin{array}{l}\text { Element free } \\
\text { Galerkin } \\
{[31]}\end{array}$ & 5.772 & 8.529 & 9.845 & \\
\hline & & $\begin{array}{l}\text { Discrete } \\
\text { Green } \\
\text { function [33] }\end{array}$ & 5.770 & 8.525 & 9.831 & \\
\hline \multirow{12}{*}{ CCCC } & \multirow{4}{*}{$\alpha=0$} & $\begin{array}{l}\text { DSCDQM- } \\
\text { RSK }\end{array}$ & 6.780 & 8.953 & 10.29 & 1.632990 \\
\hline & & $M L S D Q M$ & 6.780 & 8.953 & 10.29 & 1.646533 \\
\hline & & $\begin{array}{l}\text { Element free } \\
\text { Galerkin } \\
{[31]} \\
\end{array}$ & 6.748 & 8.905 & 10.24 & \\
\hline & & $\begin{array}{l}\text { Discrete } \\
\text { Green } \\
\text { function [33] }\end{array}$ & 6.780 & 8.953 & 10.29 & \\
\hline & \multirow{4}{*}{$\alpha=0.4$} & $\begin{array}{l}\text { DSCDQM- } \\
\text { RSK }\end{array}$ & 7.402 & 9.770 & 11.23 & 1.716354 \\
\hline & & $M L S D Q M$ & 7.402 & 9.770 & 11.23 & 1.726170 \\
\hline & & $\begin{array}{l}\text { Element free } \\
\text { Galerkin } \\
{[31]}\end{array}$ & 7.371 & 9.723 & 11.18 & \\
\hline & & $\begin{array}{l}\text { Discrete } \\
\text { Green } \\
\text { function [33] }\end{array}$ & 7.402 & 9.770 & 11.23 & \\
\hline & \multirow{4}{*}{$\alpha=0.8$} & $\begin{array}{l}\text { DSCDQM- } \\
\text { RSK }\end{array}$ & 7.945 & 10.48 & 12.05 & 1.731625 \\
\hline & & $M L S D Q M$ & 7.945 & 10.48 & 12.05 & 1.742609 \\
\hline & & $\begin{array}{l}\text { Element free } \\
\text { Galerkin } \\
\text { [31] } \\
\end{array}$ & 7.915 & 10.43 & 12.01 & \\
\hline & & $\begin{array}{l}\text { Discrete } \\
\text { Green } \\
\text { function [33] }\end{array}$ & 7.945 & 10.48 & 12.05 & \\
\hline
\end{tabular}

TABLE. 7 Comparison between the natural frequency due to DSCDQM-RSK and MLSDQM with the variable thickness $\alpha, \beta$ in two direction, different boundary conditions and the previous results for a squared plate: $\left(\mathrm{h}_{0} / \mathrm{a}=0.01, \mathrm{E}_{1} / \mathrm{E}_{2}=2.45\right.$, $v=0.23, \mathrm{~K}_{1}=0, \mathrm{~K}_{2}=0, \mathrm{~K}_{3}=0$ ). 


\begin{tabular}{|c|c|c|c|c|c|c|}
\hline \multirow{3}{*}{$\begin{array}{l}\text { Boundary } \\
\text { coditions }\end{array}$} & \multicolumn{3}{|c|}{ natural frequency } & \multirow{3}{*}{$\overline{\omega_{1}}$} & \multirow{3}{*}{$\overline{\omega_{2}}$} & \multirow{3}{*}{$\overline{\omega_{3}}$} \\
\hline & \multicolumn{2}{|c|}{$\begin{array}{l}\text { variable } \\
\text { thickness }\end{array}$} & \multirow[t]{2}{*}{ results } & & & \\
\hline & $\alpha$ & $\beta$ & & & & \\
\hline \multirow{16}{*}{ SSSS } & \multirow{4}{*}{-0.5} & \multirow{4}{*}{-0.5} & $\begin{array}{l}\text { DSCDQM- } \\
\text { RSK }\end{array}$ & 3.635 & 5.335 & 6.087 \\
\hline & & & $M L S D Q M$ & 3.635 & 5.335 & 6.087 \\
\hline & & & $\begin{array}{l}\text { Element free } \\
\text { Galerkin } \\
{[31]} \\
\end{array}$ & 3.633 & 5.3460 & 6.0957 \\
\hline & & & $\begin{array}{l}\text { Discrete } \\
\text { Green } \\
\text { function [33] }\end{array}$ & 3.635 & 5.335 & 6.087 \\
\hline & \multirow{4}{*}{-0.5} & \multirow{4}{*}{0.5} & $\begin{array}{l}\text { DSCDQM- } \\
\text { RSK }\end{array}$ & 4.704 & 6.937 & 7.966 \\
\hline & & & $M L S D Q M$ & 4.704 & 6.937 & 7.966 \\
\hline & & & $\begin{array}{l}\text { Element free } \\
\text { Galerkin } \\
{[31]}\end{array}$ & 4.707 & 6.9422 & 7.9751 \\
\hline & & & $\begin{array}{l}\text { Discrete } \\
\text { Green } \\
\text { function [33] }\end{array}$ & 4.704 & 6.937 & 7.966 \\
\hline & \multirow{4}{*}{0.5} & \multirow{4}{*}{-0.5} & $\begin{array}{l}\text { DSCDQM- } \\
\text { RSK }\end{array}$ & 4.708 & 6.933 & 7.904 \\
\hline & & & $M L S D Q M$ & 4.708 & 6.933 & 7.904 \\
\hline & & & $\begin{array}{l}\text { Element free } \\
\text { Galerkin } \\
{[31]}\end{array}$ & 4.708 & 6.9420 & 7.9146 \\
\hline & & & $\begin{array}{l}\text { Discrete } \\
\text { Green } \\
\text { function [33] }\end{array}$ & 4.708 & 6.933 & 7.904 \\
\hline & \multirow{4}{*}{0.5} & \multirow{4}{*}{0.5} & $\begin{array}{l}\text { DSCDQM- } \\
\text { RSK }\end{array}$ & 6.086 & 9.022 & 10.350 \\
\hline & & & $M L S D Q M$ & 6.086 & 9.022 & 10.350 \\
\hline & & & $\begin{array}{l}\text { Element free } \\
\text { Galerkin } \\
\text { [31] } \\
\end{array}$ & 6.099 & 9.013 & 10.359 \\
\hline & & & $\begin{array}{l}\text { Discrete } \\
\text { Green } \\
\text { function [33] }\end{array}$ & 6.086 & 9.022 & 10.350 \\
\hline \multirow{16}{*}{$\mathrm{CCCC}$} & \multirow{4}{*}{-0.5} & \multirow{4}{*}{-0.5} & $\begin{array}{l}\text { DSCDQM- } \\
\text { RSK }\end{array}$ & 4.955 & 6.548 & 7.440 \\
\hline & & & $M L S D Q M$ & 4.955 & 6.548 & 7.440 \\
\hline & & & $\begin{array}{l}\text { Element free } \\
\text { Galerkin } \\
{[31]}\end{array}$ & 4.936 & 6.532 & 7.4167 \\
\hline & & & $\begin{array}{l}\text { Discrete } \\
\text { Green } \\
\text { function [33] }\end{array}$ & 4.955 & 6.548 & 7.440 \\
\hline & \multirow{4}{*}{-0.5} & \multirow{4}{*}{0.5} & $\begin{array}{l}\text { DSCDQM- } \\
\text { RSK }\end{array}$ & 6.453 & 8.510 & 9.748 \\
\hline & & & $M L S D Q M$ & 6.453 & 8.510 & 9.748 \\
\hline & & & $\begin{array}{l}\text { Element free } \\
\text { Galerkin } \\
{[31]} \\
31]\end{array}$ & 6.427 & 8.481 & 9.715 \\
\hline & & & $\begin{array}{l}\text { Discrete } \\
\text { Green } \\
\text { function [33] }\end{array}$ & 6.453 & 8.510 & 9.748 \\
\hline & \multirow{4}{*}{0.5} & \multirow{4}{*}{-0.5} & $\begin{array}{l}\text { DSCDQM- } \\
\text { RSK }\end{array}$ & 6.447 & 8.525 & 9.671 \\
\hline & & & $M L S D Q M$ & 6.447 & 8.525 & 9.671 \\
\hline & & & $\begin{array}{l}\text { Element free } \\
\text { Galerkin } \\
{[31]}\end{array}$ & 6.420 & 8.501 & 9.639 \\
\hline & & & $\begin{array}{l}\text { Discrete } \\
\text { Green } \\
\text { function } \\
{[33]}\end{array}$ & 6.45 & 8.525 & 9.671 \\
\hline & \multirow{4}{*}{0.5} & \multirow{4}{*}{0.5} & $\begin{array}{l}\text { DSCDQM- } \\
\text { RSK } \\
\end{array}$ & 8.390 & 11.076 & 12.666 \\
\hline & & & $M L S D Q M$ & 8.390 & 11.076 & 12.666 \\
\hline & & & $\begin{array}{l}\text { Element free } \\
\text { Galerkin } \\
\text { [31] } \\
\end{array}$ & 8.36 & 11.040 & 12.632 \\
\hline & & & $\begin{array}{l}\text { Discrete } \\
\text { Green } \\
\text { function [33] }\end{array}$ & 8.390 & 11.076 & 12.666 \\
\hline
\end{tabular}

Furthermore, a parametric study is introduced to investigate the influence of elastic and geometric characteristics of the composite on the values of natural frequencies.

Figures (3-15) show that the natural frequencies decrease with increasing variable thickness $(\alpha, \beta)$, Young's modulus gradation ratio and thickness ratio. As well as, Figs. (310,12-13) show that the natural frequencies are increased with increasing variation parameter of foundation stiffness $\gamma$, Winkler and Pasternak foundation, shear modulus gradation ratio and aspect ratio $\mathrm{a} / \mathrm{b}$. The case of $\left(\mathrm{E}_{1}=\mathrm{E}_{2}=\mathrm{E}_{3}=\mathrm{E}_{4}=\mathrm{E}_{5}\right.$, $\mathrm{G}_{1}=\mathrm{G}_{2}=\mathrm{G}_{3}=\mathrm{G}_{4}=\mathrm{G}_{5}$ and $\mathrm{h}_{1}=\mathrm{h}_{2}=\mathrm{h}_{3}=\mathrm{h}_{4}=\mathrm{h}_{5}$ ) is a limiting case of this study which was previously solved in [28] and [29-33]. Figures(12-15) show the first three mode shapes of the vibration waves along the interface.Furthermore, Figs.(1415) show that the amplitudes of $\mathrm{W}$ increase with increasing linear and nonlinear elastic foundation parameters.Also, these figures show that the natural frequencies do not affect significantly by nonlinear elastic foundation parameter $\mathrm{k}_{3}$.

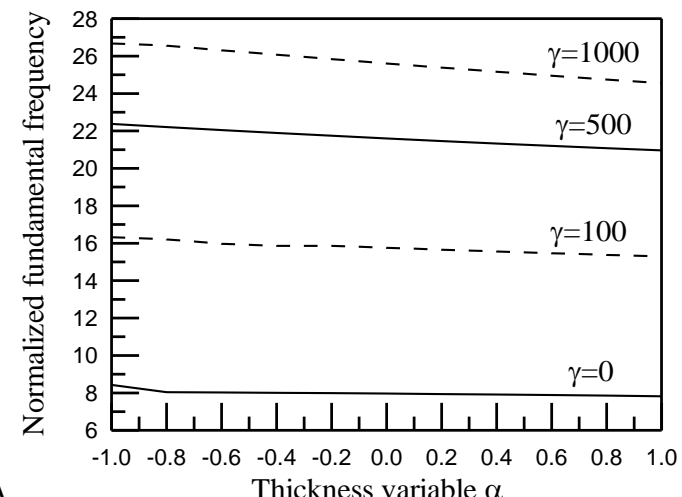

A Thickness variable $\alpha$

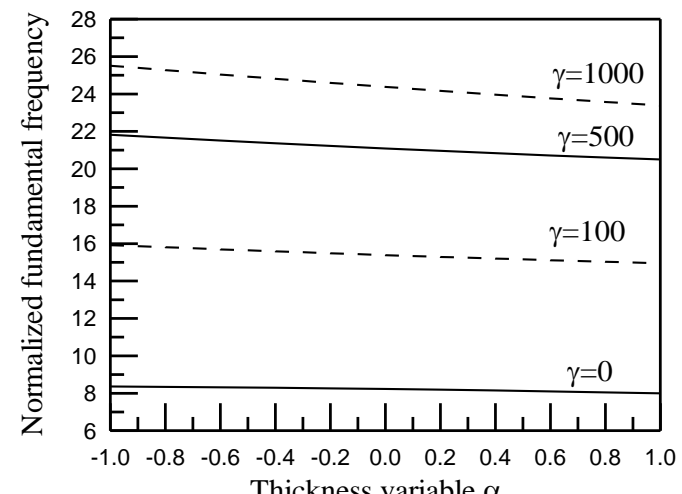

B

FIG. 3 Variation of the normalized fundamental frequency withthickness variable $(\alpha, \beta)$ and non-uniform Winkler foundationfor squared simply supported plates. $A$ : $\beta=0, B$ : $\beta=0.5\left(K_{1}=1000, K_{2}=0, K_{3}=0\right)$. 


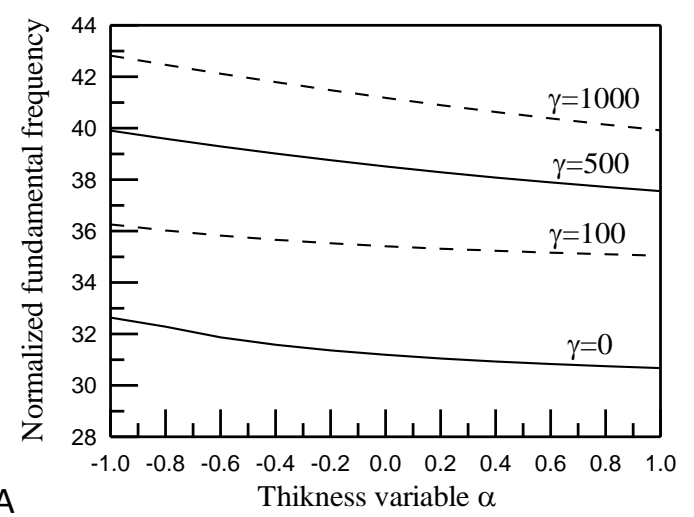

A

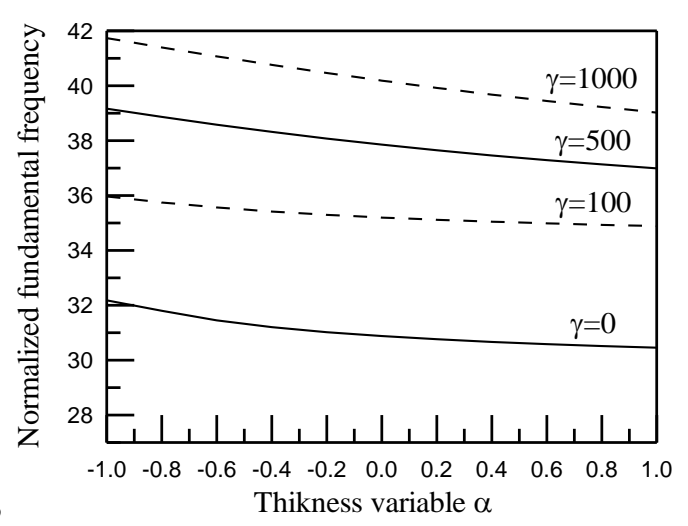

FIG. 4 Variation of the normalized fundamental frequency with thickness variable $(\alpha, \beta)$ and non-uniform Winkler and uniform Pasternak foundation for squared simply supported plates. $A$ : $\beta=0$, B: $\beta=0.5\left(K_{1}=1000\right.$, $\mathrm{K}_{2}=10, \mathrm{~K}_{3}=0$ )

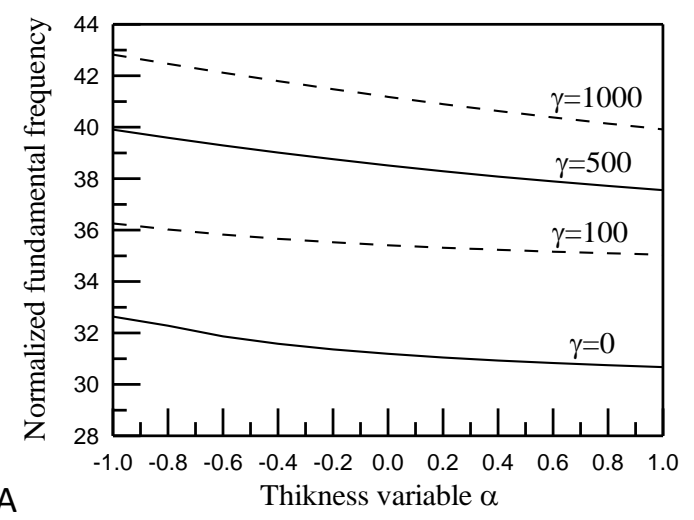

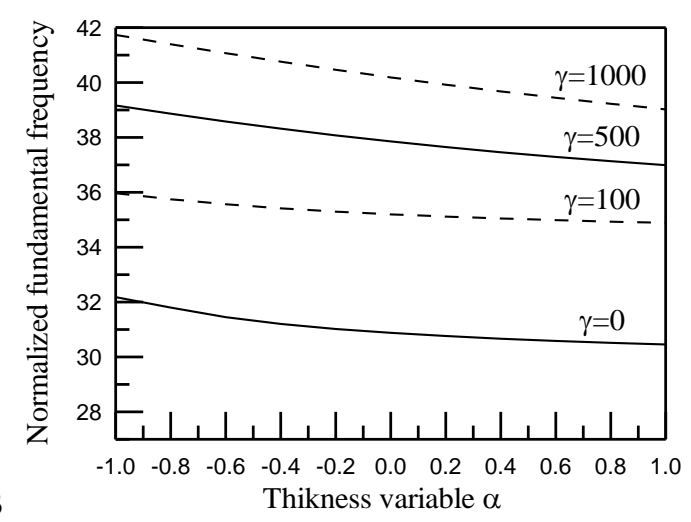

FIG. 5 Variation of the normalized fundamental frequency with thickness variable $(\alpha, \beta)$ ,non-uniform ,nonlinear Winkler and Pasternak foundation for squared simply supported plates. $A: \beta=0, B: \beta=0.5$ $\left(\mathrm{K}_{1}=1000, \mathrm{~K}_{2}=10, \mathrm{~K}_{3}=50\right)$
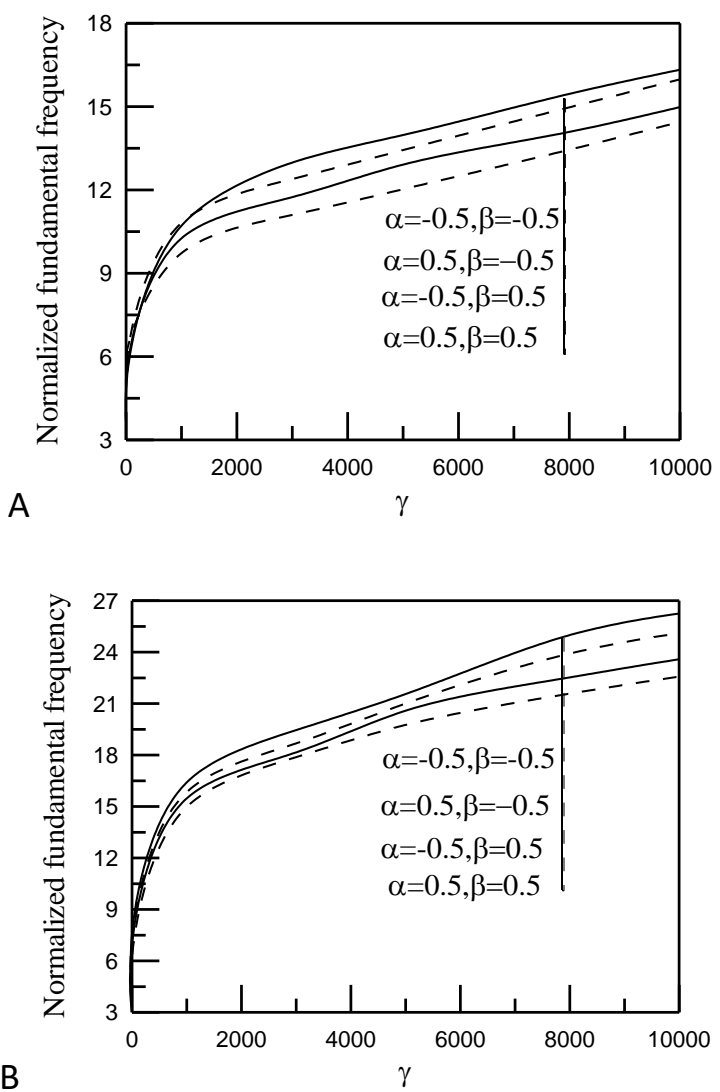

FIG. 6 Variation of the normalized fundamental frequency with thickness variable $(\alpha, \beta)$ and non-uniform Winkler foundation for squared simply supported plates . A: $\mathrm{K}_{1}=10, \mathrm{~B}$ : $\mathrm{K}_{1}=100 \quad\left(\mathrm{~K}_{2}=0, \mathrm{~K}_{3}=0\right)$ 

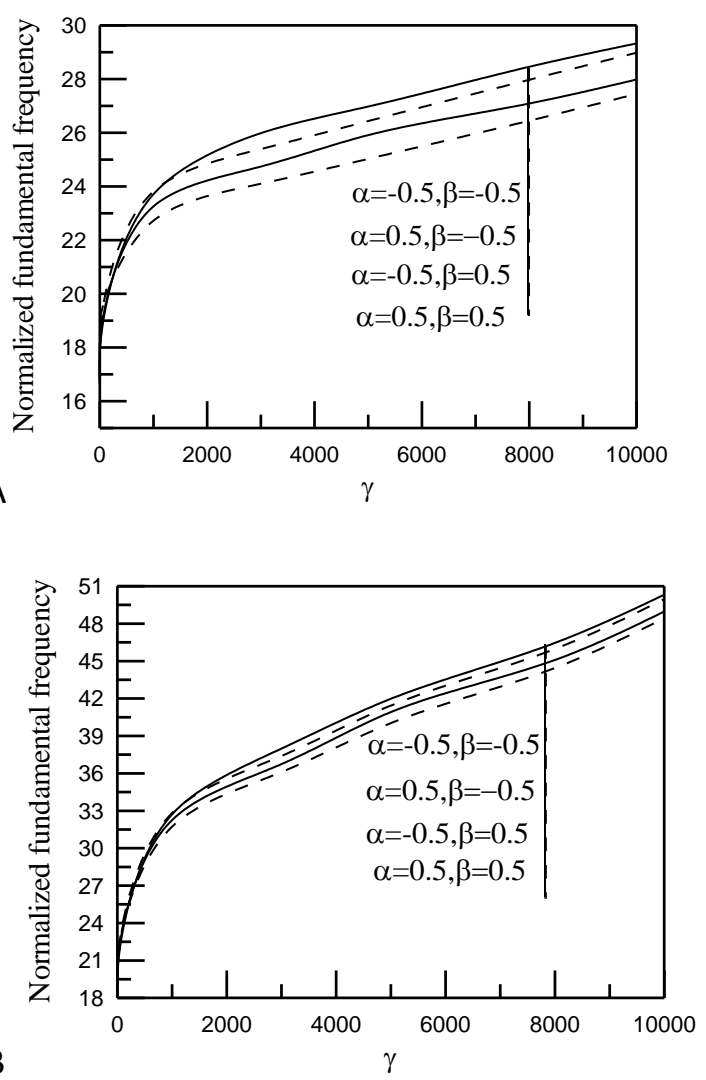

FIG. 7 Variation of the normalized fundamental frequency with thickness variable $(\alpha, \beta)$ and non-uniform Winkler and Pasternak foundation for squared simply supported plates. A: $\mathrm{K}_{1}=10, \mathrm{~B}: \mathrm{K}_{1}=100$ $\left(\mathrm{K}_{2}=10, \mathrm{~K}_{3}=0\right)$

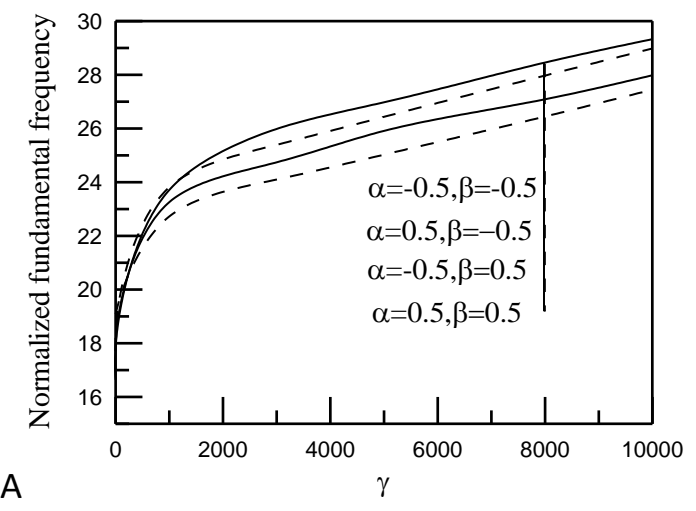

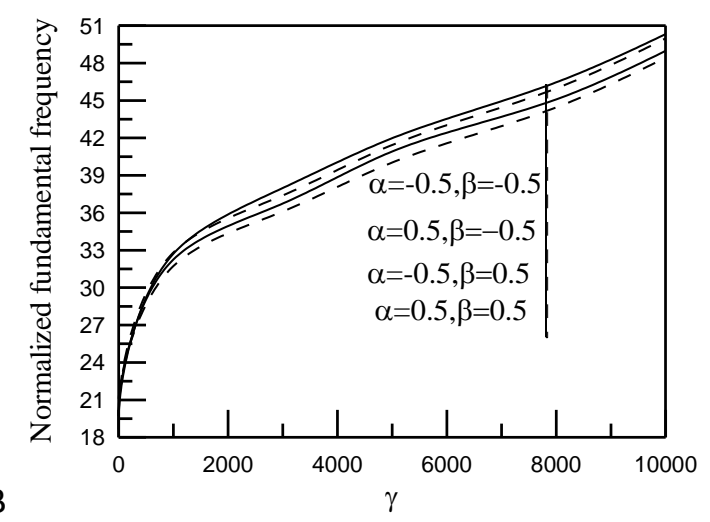

FIG. 8 Variation of the normalized fundamental frequency with thickness variable $(\alpha, \beta)$ and non-uniform, nonlinear Winkler and Pasternak foundation for squared simply supported plates. A: $\mathrm{K}_{1}=10$, B: $\mathrm{K}_{1}=100$ ( $\mathrm{K}_{2}=10, \mathrm{~K}_{3}=100$ )
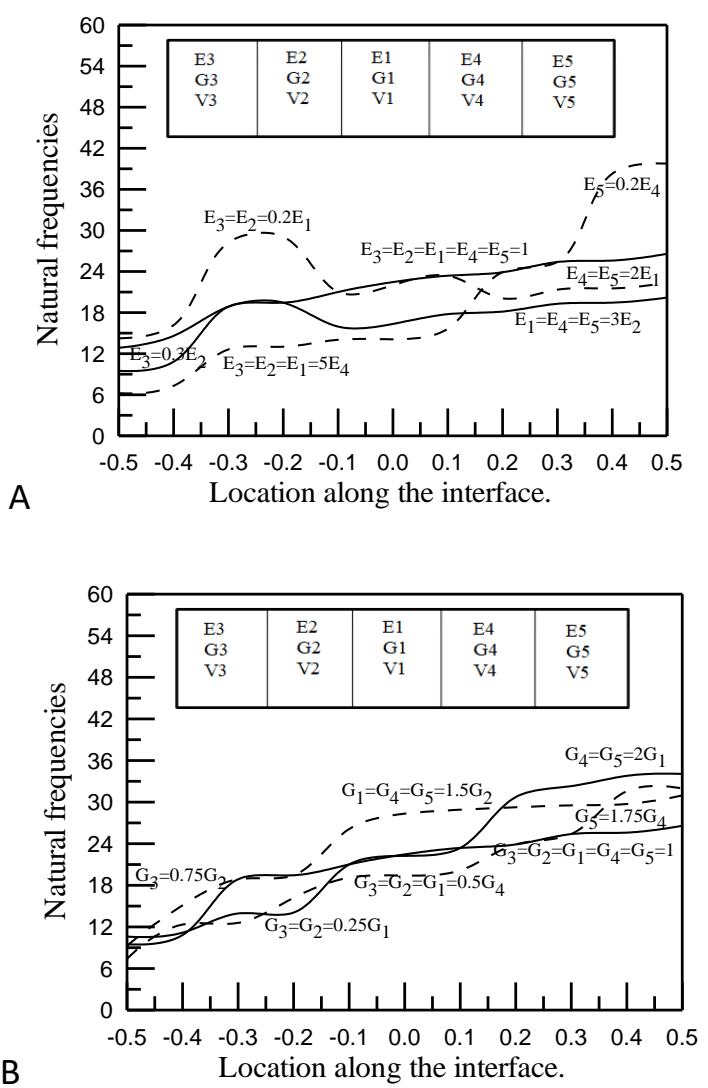

FIG. 9 Variation of the natural frequencies with

Young's and Shear modulus gradation ratio of a squared simply supported composite $\left(\mathrm{K}_{1}=100, \mathrm{~K}_{2}=10, \mathrm{~K}_{3}=50, \mathrm{~h}_{0} / \mathrm{a}=0.01, \gamma=1000\right.$, $\alpha=-0.5, \beta=-0.5, \mathrm{v}_{1}=\mathrm{v}_{2}=\mathrm{v}_{3}=\mathrm{v}_{4}=\mathrm{v}_{5}$ ). 
A
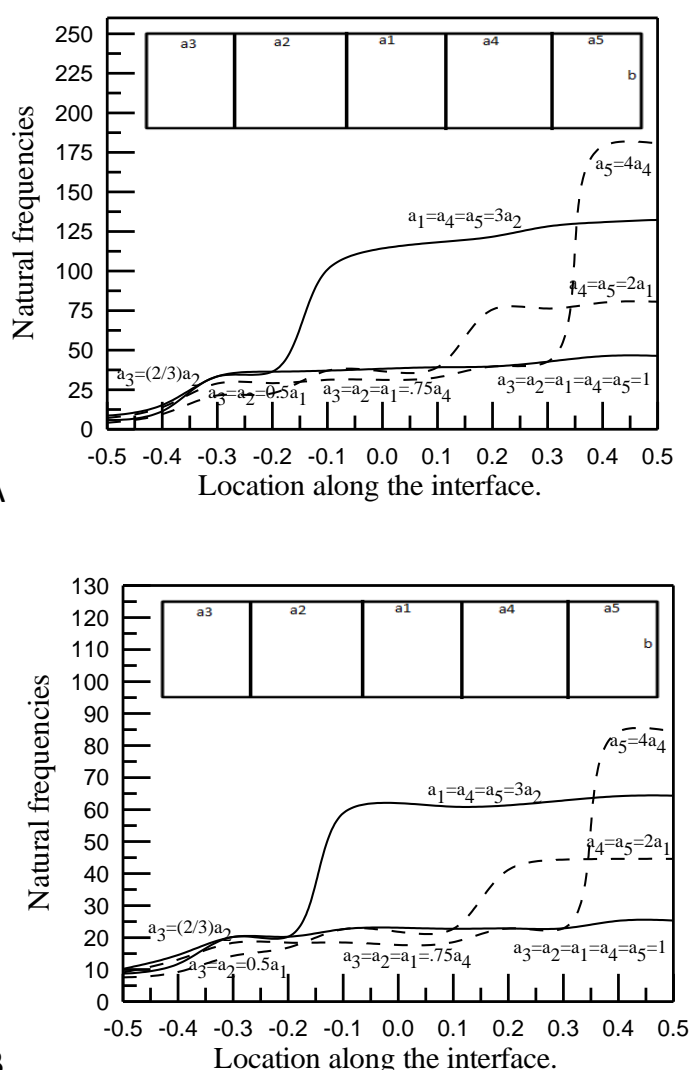

FIg. 10 Variation of the natural frequencies with aspect ratio $(\mathrm{a} / \mathrm{b})$ and thickness (h/a) for Clamped composite plates. A: $\mathrm{h}_{0} / \mathrm{a}=0.01, \mathrm{~B}: \mathrm{h}_{0} / \mathrm{a}=0.1 \quad\left(\mathrm{~K}_{1}=500\right.$ $\mathrm{K}_{2}=100, \mathrm{~K}_{3}=100, \gamma=1000, \alpha=-0.5, \beta=-$ $0.5 ; \mathrm{E}_{1}=\mathrm{E}_{2}=\mathrm{E}_{3}=\mathrm{E}_{4}=\mathrm{E}_{5}, \mathrm{G}_{1}=\mathrm{G}_{2}=\mathrm{G}_{3}=\mathrm{G}_{4}=\mathrm{G}_{5}$, $\mathrm{v}_{1}=\mathrm{v}_{2}=\mathrm{v}_{3}=\mathrm{v}_{4}=\mathrm{v}_{5}$ ).

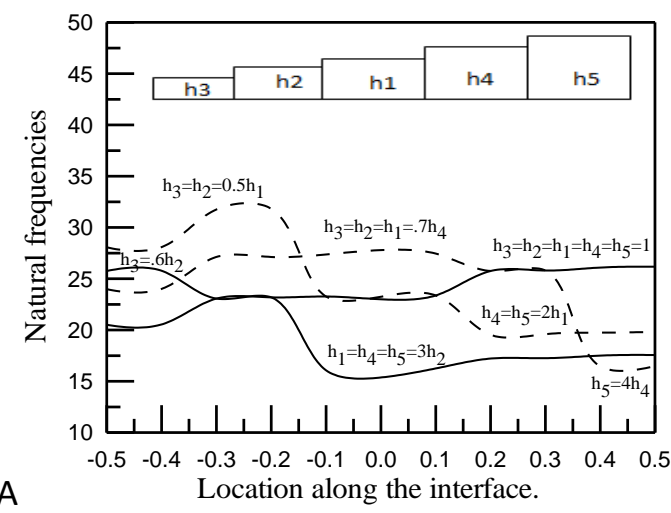

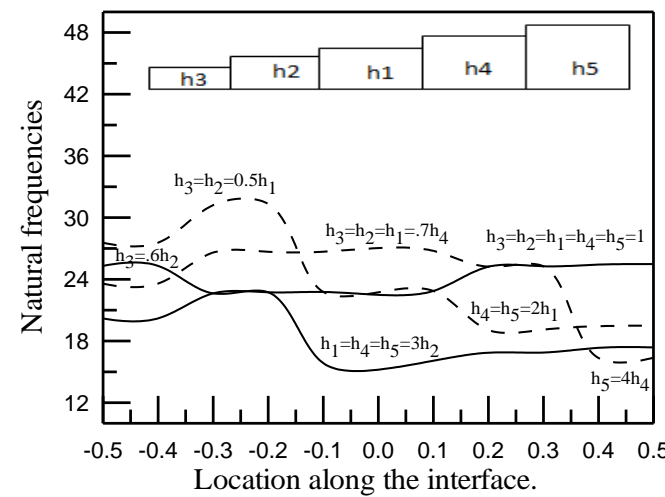

FIG. 11 Variation of the natural frequencies with thickness of a squared elastically supported composite. A: Simply supported plates, B: Clamped plates $\left(\mathrm{K}_{1}=1000, \mathrm{~K}_{2}=1000, \mathrm{~K}_{3}=1000, \gamma=1000\right.$, $\alpha=-0.5, \beta=-0.5 ; E_{1}=E_{2}=E_{3}=E_{4}=E_{5}$, $\left.\mathrm{G}_{1}=\mathrm{G}_{2}=\mathrm{G}_{3}=\mathrm{G}_{4}=\mathrm{G}_{5}, \mathrm{v}_{1}=\mathrm{v}_{2}=\mathrm{v}_{3}=\mathrm{v}_{4}=\mathrm{v}_{5}\right)$.
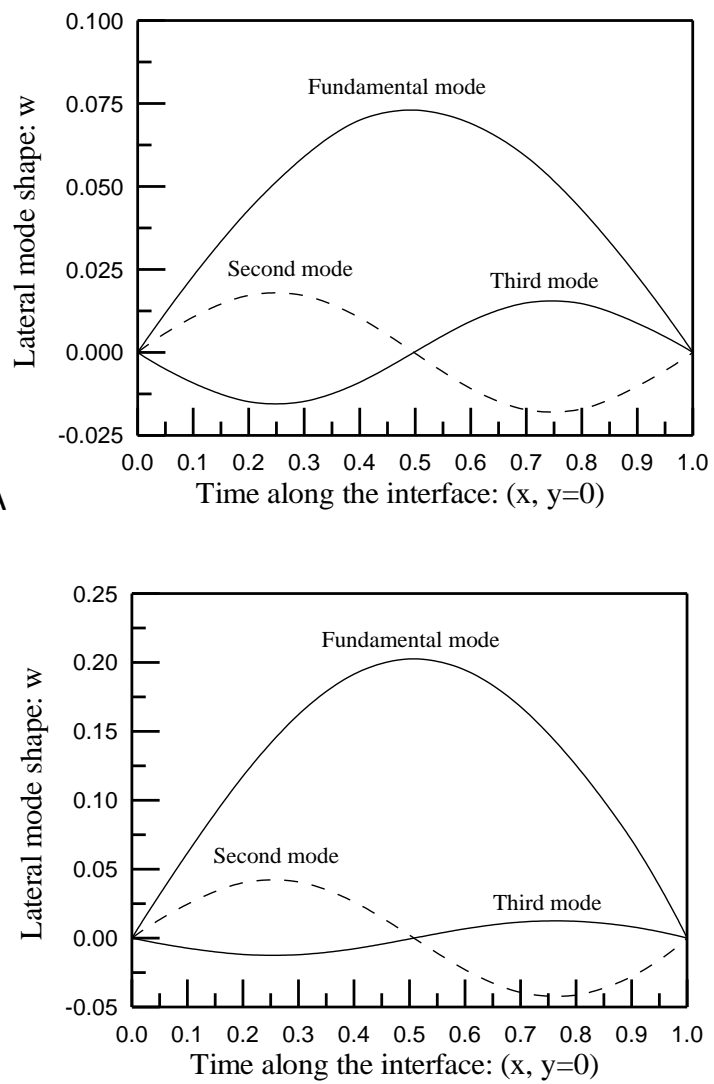

Fig. 12 Variation of the lateral mode shapes with time and non-uniform Winkler foundation for squared clamped plate at $\alpha=0$, $\beta=0, K_{2}=0, K_{3}=0, \gamma=1000$. A: $K_{1}=10$, B: $K_{1}=100$ 

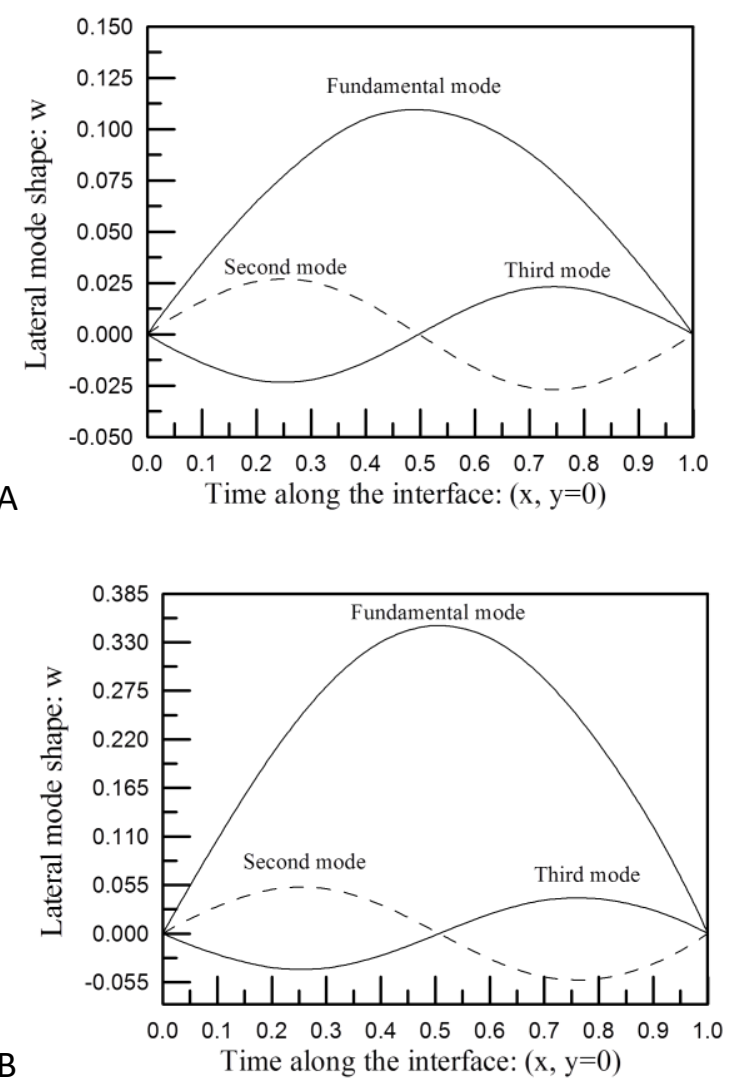

FIG. 13 Variation of the lateral mode shapes with time and non-uniform Winkler and Pasternak foundation for squared clamped plate at $\alpha=-0.5$, $\beta=0.5, K_{2}=100, K_{3}=0, \gamma=1000 . A: K_{1}=10$, B: $\mathrm{K}_{1}=500$

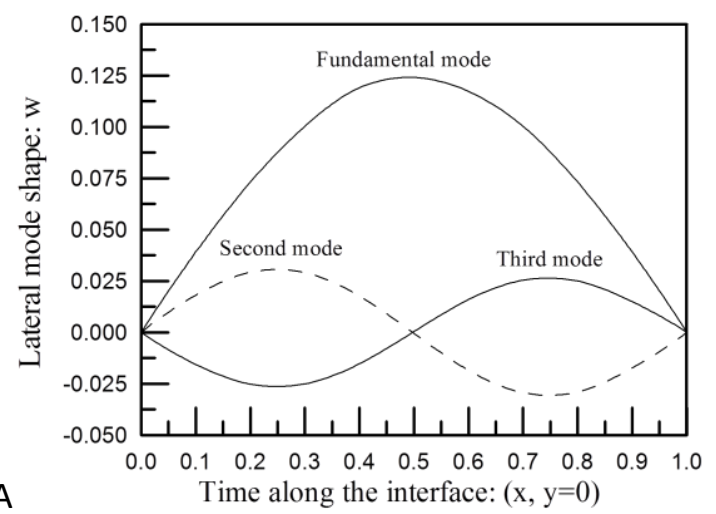

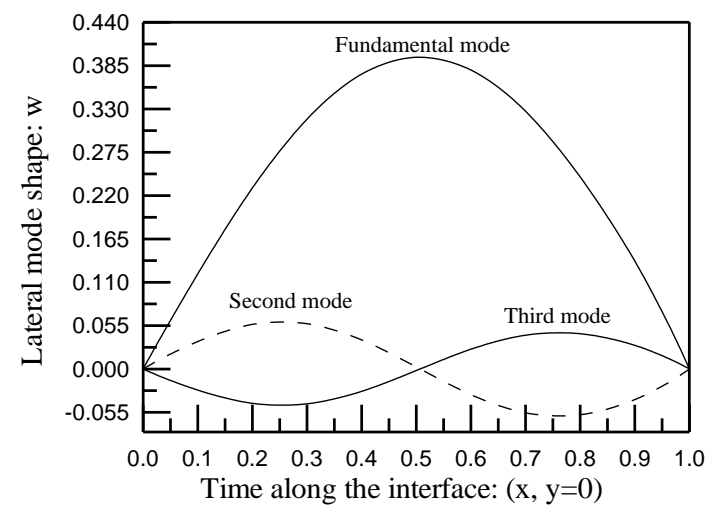

Fig. 14 Variation of the lateral mode shapes with time and non-uniform and nonlinear Winkler foundation for squared clamped plate at $\alpha=-0.5, \beta=0.5, K_{2}=0, K_{3}=100$, $\gamma=1000$. A: $K_{1}=10, B: K_{1}=500$
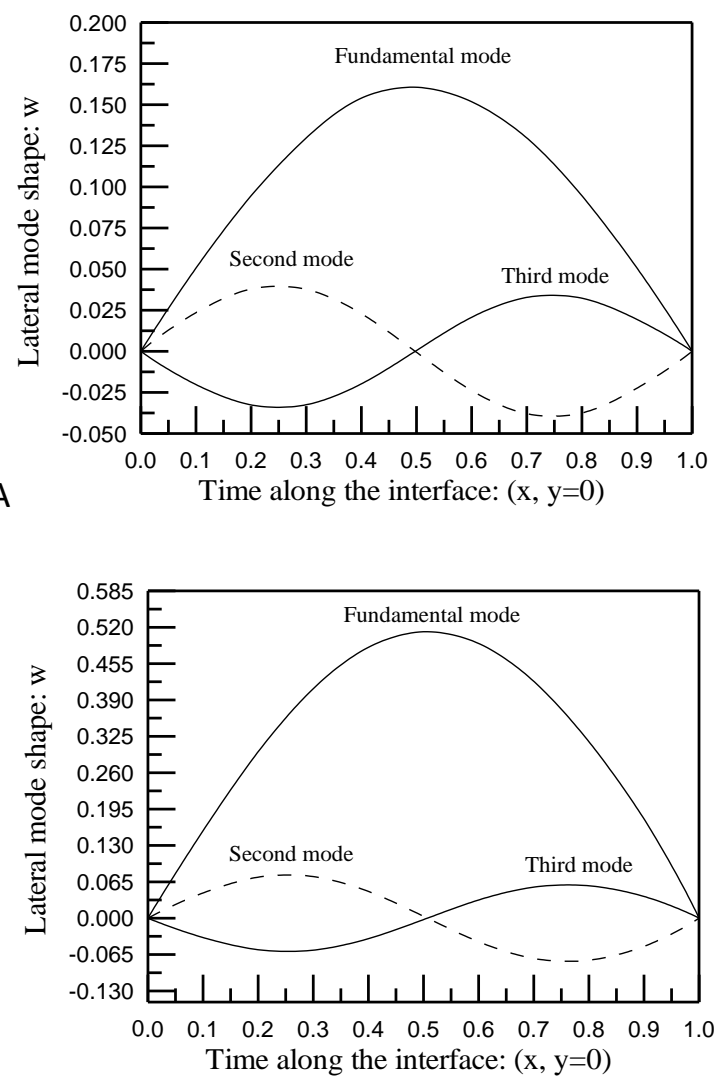

Fig. 15 Variation of the lateral mode shapes with time and non-uniform and nonlinear Winkler and Pasternak foundation for squared clamped plate at $\alpha=-0.5$, $\beta=0.5, K_{2}=100, K_{3}=100, \gamma=1000$. A: $K_{1}=10, B: K_{1}=500$ 


\section{CONCLUSION}

Different Quadrature schemes have been successfully applied for vibration analysis of composite plate with variable thickness resting on non-uniform and nonlinear elastic foundation. Iterative quadrature technique is used to solve the nonlinear algebraic system. A matlab program is designed for each scheme such that the maximum error (comparing with the previous exact results) is $\leq 10^{-10}$. Also, Execution time for each scheme, is determined. It is concluded that discrete singular convolution differential quadrature method based on regularized Shannon kernel (DSCDQM-RSK) with grid size $\geq 13 * 13$, bandwidth $2 \mathrm{M}+1$ $\geq 11$ and regulization parameter $\sigma=2.82 \mathrm{hx}$ leads to best accurate efficient results for the concerned problem. shear factor correction $(\mathrm{K}=5 / 6-\mathrm{v})$ is considered for such problem. Based on this scheme, a parametric study is introduced to investigate the influence of elastic and geometric characteristics of the vibrated plate, on results. It is aimed that these results may be useful for design purposes of engineering fields.

\section{REFERENCES}

[1] Y. P. Xu , D. Zhou, Three-dimensional elasticity solution for simply supported rectangular plates with variable thickness, Journal of Strain Analysis for Engineering Design, 43 (2008)165-

[2] Y. Xu, D. Zhou, K. Liu, Three-dimensional thermo elastic analysis of rectangular plates with variable thickness subjected to thermomechanical loads, Journal of Thermal Stresses, 33 (2010) 1136- 1155.

[3] A.H. Sofiyev, H.M. Halilov, N. Kuruoglu, Analytieal solution of the dynamic behavior of non-homogenous orthotropic cylindrical shells on elastic foundations under moving loads, Journal of Engineering Mathematics , 69(2011) 359-371.

[4] D. Shi, H. Zhang, Q. Wang, S. Zha, Free and Forced Vibration of the Moderately Thick Laminated Composite Rectangular Plate on Various Elastic Winkler and Pasternak Foundations, Shock and Vibration, 2017 (2017) 1-24

[5] K.K. Viswanathan, P.V. Navaneethakrishnan , Z. A. Aziz, Buckling Analysis of Rectangular Plates with Variable Thickness Resting on Elastic Foundation, Earth and Environmental Science, 23 (2015) 1-8.

[6] K.K. Viswanathan, J. Saira, A. A. Zainal, Free Vibration of Symmetric Angle-Ply Laminated Conical Shell Frusta of Variable Thickness including Shear Deformation Theory by Spline Method. Structural Engineering \& Mechanics, An International Journal, 45 (2013) 259275.

[7] S. Chakraverty, K. K. Pradhan, Free Vibration Of Functionally Graded Thin Rectangular Plates Resting On Winkler Elastic Foundation With General Boundary Conditions Using Rayleigh-Ritz Method, Int. J. Appl. Mechanics, 6(2014).

[8] A. Khanna, A. K. Sharma, Vibration Analysis Of Visco- Elastic Square Plate Of Variable Thickness With Thermal Gradient, International Journal of Engineering and Applied Sciences (IJEAS), 3 (2011) 1-6

[9] L. Chen, Y.M. Cheng, The complex variable reproducing kernel particle method for bending problems of thin plates on elastic foundations, Computational Mechanics, 62, (2018) 67-80.

[10] M. Huang, J. Ma, J. N. Tang, H. Yuan, Mixed boundary node method for free vibration analysis of rectangular plates with variable thickness and general boundary conditions, WIT Transactions on Modelling and Simulation, 56(2013).

[11] A. S. Ashour, vibration of variable thickness plates with edges elastically restrained against rotational and translation,Thin-Walled Structures, 42(2004) 1-24.

[12] H. Zafarmand, M. Kadkhodayan, Free vibration analysis of thick disks with variable thickness containing orthotropic and nonhomogeneous material using finite element method, Journal of theoretical and applied mechanics , 53(2015) 1005-1018.

[13] V. Tahouneh, Free vibration analysis of bidirectional functionally graded annular plates resting on elastic foundations using differential quadrature method, Structural Engineering and Mechanics, 52(2014) 663-686.
[14] R. Saini, R. Lal, Transverse Vibration of Non-Homogeneous Rectangular Plates of Variable Thickness Using GDQ, International Journal of Computer and Information Engineering, 8(2014)11971202

[15] A. G. Arani, M. Khani , Z. K. Maraghi, Dynamic analysis of a rectangular porous plate resting on an elastic foundation using highorder shear deformation theory, Journal of Vibration and Control 24, (2018) 3713-3698.

[16] O.Ragb, M.Mohamed, M.S. Matbuly, Free Vibration of a Piezoelectric Nanobeam Resting on Nonlinear Winkler-Pasternak Foundation by Quadrature Methods, Heliyon, 5(2019) e01856.

[17] O.Ragb, M.Mohamed, M.S. Matbuly , Vibration Analysis of Magneto-Electro-Thermo NanoBeam Resting on Nonlinear Elastic Foundation Using Sinc and Discrete Singular Convolution Differential Quadrature Method, Modern Applied Science, 13(2019)

[18] O.Ragb, M. salah, M.S. Matbuly, R.M. Amer, Vibration analysis of piezoelectric composite using Sinc and Discrete Singular Convolution differential quadrature techniques, Journal of Engineering and Applied Sciences, (2019)6540-6553.

[19] A. Sayyidmousavi, M. Foroutan, Z. Fawaz, Free Vibration Analysis of Functionally Graded Nanocomposite Beams on Elastic Foundation Using a Mesh-Free Method, Latin American Journal of Solids and Structures, 14 (2017) 2107-2122.

[20] O. Ragb, M.S. Matbuly, M. Nassar, Analysis Of Composite plates Using Moving Least Squares Differential Quadrature Method,Applied Mathematics and Computation, , 238(2014) 225-236.

[21] R.M. Dastjerdi, G. Payganeh , S.R.Mirakabad, M. J. M.Taheri, Static and Free Vibration Analyses of Functionally Graded Nano composite Plates Reinforced by Wavy Carbon Nanotubes Resting on a Pasternak Elastic Foundation, Mechanics of Advanced Composite Structures, 3 (2016) 123-135.

[22] V.Panc. , Theory of elastic plates, Leydon : Noordhoff International Publishing, (1975) 13-41.

[23] J.N. Reddy, Mechanics of Laminated Composite Plates. Theory and Analysis, CRC Press, Boca Raton, FL(1997).

[24] J.N. Reddy, Theory and analysis of elastic plates, Taylor\& Francis: Philadelphia, PA(1999).

[25] V. Birman , C. W. Bert, On the Choice of Shear Correction Factor in Sandwich Structures, Journal of Sandwich Structures and Materials 4 (2002) 83-95.

[26] V.Birman , C. W. Bert, Effect of Shear Correction Factor on Response of Cross-ply Laminated Plates using FSDT, Defence Science Journal, 55 (2005) 377-387.

[27] F. Gruttmann, W. Wagner, Shear correction factors for layered plates and shells,Comput Mech, 59 (2017) 129-146.

[28] Y. Xiang, C. M. Wang, S. Kitipornchai, Exact vibration solution fo initially stressed Mindlin plates on Pasternak foundation, International Journal of Mechanic Science, 36 , (1994) 311-316.

[29] D. Zhou, Y. K. Cheung, S. H. Lo , F. T. K. Au, Three dimensional vibration analysis of rectangular thick plates on Pasternak foundation, International Journal of Numerical Methods in Engineering, 59 (2004) 1313-1334.

[30] J. M. Ferreira, C. M. C. Roque, A. M. A. Neves, R. M. N. Jorge , C. M. M. Soares, Analysis of plates on Pasternak foundations by radial basis functions, Computational Mechanics, 46(2010)791-803.

[31] E. Bahmyari, A. R. Ranji, Free vibration analysis of orthotropic plates with variable thickness resting on non-uniform elastic foundation by element free Galerkin method,Journal of Mechanical Science and Technology, 26 (2012) 2685-2694.

[32] M. H. Omurtag, A.Ozutok, A. Y. Akoz, Free vibration analysis of Kirchhoff plates resting on elastic foundation by mixed finite element formulation based on gateaux differential, International Journal of Numerical Methods in Engineering, 40 (1997) 295-317.

[33] M. Huang, X. Q. Ma, T.Sakiyama, H. Matuda, C. Morita, Free vibration analysis of orthotropic rectangular plates with variable thickness and general boundary conditions, Journal of Sound and Vibration, 288(2005) 931-955.

[34] O. Ragb, L. F. Seddek, M. S. Matbuly, Iterative differential quadrature solutions for Bratu problem, Computers \& Mathematics with Applications , 74, 2 (2017) 249-257. 